\title{
Zoonotic MERS-CoV transmission: modeling, backward bifurcation and optimal control analysis
}

\author{
Indrajit Ghosh • Sk Shahid Nadim 1 \\ Joydev Chattopadhyay
}

Received: 19 October 2020 / Accepted: 27 January 2021 / Published online: 8 February 2021

(C) The Author(s), under exclusive licence to Springer Nature B.V. part of Springer Nature 2021

\begin{abstract}
Middle East Respiratory Syndrome Coronavirus (MERS-CoV) can cause mild to severe acute respiratory illness with a high mortality rate. As of January 2020, more than 2500 cases of MERS-CoV resulting in around 860 deaths were reported globally. In the absence of neither effective treatment nor a readyto-use vaccine, control measures can be derived from mathematical models of disease epidemiology. In this manuscript, we propose and analyze a compartmental model of zoonotic MERS-CoV transmission with two co-circulating strains. The human population is considered with eight compartments while the zoonotic camel population consist of two compartments. The expression of basic reproduction numbers are obtained for both single strain and two strain version of the proposed model. We show that the disease-free equilibrium of the system with single stain is globally asymptotically stable under some parametric conditions. We also demonstrate that both models undergo backward bifurcation phenomenon, which in turn indicates that only keeping $R_{0}$ below unity may not ensure eradica-
\end{abstract}

I. Ghosh

Department of Computational and Data Sciences, Indian Institute of Science, Bengalore, Karnataka 560012, India e-mail: indra7math@gmail.com

Sk. S. Nadim $(\varangle) \cdot$ J. Chattopadhyay

Agricultural and Ecological Research Unit, Indian

Statistical Institute, Kolkata 700 108, India

e-mail: nadimskshahid@gmail.com

J. Chattopadhyay

e-mail: joydev@isical.ac.in tion. To the best of the authors knowledge, backward bifurcation was not shown in a MERS-CoV transmission model previously. Further, we perform normalized sensitivity analysis of important model parameters with respect to basic reproduction number of the proposed model. Furthermore, we perform optimal control analysis on different combination interventions with four components namely preventive measures such as use of masks, isolation of strain-1 infected people, strain-2 infected people and infected camels. Optimal control analysis suggests that combination of preventive measures and isolation of infected camels will eventually eradicate the disease from the community.

Keywords MERS-CoV · Global stability analysis . Backward bifurcation - Optimal control analysis

\section{Introduction}

An outbreak of Middle East respiratory syndrome coronavirus (MERS-CoV) is ongoing in the Arabian Peninsula, with the first case identified in Jeddah, Saudi Arabia, in June 2012 [36]. Phylogenetic analyses have indicated that the virus emerged in July 2011, with a broad uncertainty range, and that the outbreak results from multiple introductions of a weakly transmissible virus that is geographically dispersed [8]. Since September 2012, WHO has been notified of more than 2500 laboratory-confirmed cases of infection with MERS$\mathrm{CoV}$ and at least 860 deaths related to MERS-CoV. 
Sporadic cases have been imported to Europe, Africa, Asia and North America via returning travelers from the Middle East, but no sustained transmission has been reported in those regions. Sporadic introductions of MERS-CoV into humans are suspected to involve bats [15] and/or camels [27] with camels implicated as the likely source of most zoonotic infections of MERS$\mathrm{CoV}$ in Saudi Arabia. Meanwhile, there is considerable uncertainty about the extent of human-to-human transmission and it is unclear whether MERS-CoV has the potential for epidemic spread. Transmission appears limited among family members but may be amplified in health care settings [8]. An understanding of the MERS-CoV epidemiology and transmission pathways are critically needed to devise effective surveillance, prevention and control strategies.

Mathematical models provide a suitable framework in this situation to understand the transmission dynamics and design of prevention strategies. There are few modelling approaches related to MERS-CoV epidemics. [6] proposed a data-driven model for MERS$\mathrm{CoV}$ transmission and they found strong support for $R_{0}<1$ ( $R_{0}$ measures the average number of secondary cases from each infected person) in the initial stage of the epidemic in Saudi Arabia. [14] used the Richards model to trace the temporal course of the South Korea MERS-CoV outbreak. In 2016, a dynamic transmission model for the 2015 MERS-CoV outbreak in the Republic of Korea was considered [19]. Later on, an SEIR type mathematical model for the MERS-CoV outbreak in South Korea was considered to perform optimal control analysis [18]. A stochastic epidemiological model of MERS-CoV with zoonotic and human-to-human transmission was considered by [26] to quantify the rates of generation of cases from those two transmission routes. However, these models were based on single a strain of MERS-CoV. There is strong evidence in the literature that there are multiple strains of MERS-CoV co-circulating in the community [5,28,38]. [29], proposed a two strain model of MERS-CoV to understand the epidemiological characteristics in three affected provinces of Saudi Arabia. They found that Riyadh province is likely to have cases from two strains. Motivated by this we consider a two strain model to have some insight into the intervention strategies. There is, however, no mathematical analysis considering two strain models of MERS-CoV as far as our knowledge is concerned. The objective of this study is to investigate the qualitative effect of both human-to-human and zoonotic disease transmission on the spread of MERSCoV. To achieve this goal, a two-strain mathematical model for MERS-CoV is proposed and analyzed. We consider transmission from camels to humans as a zoonotic infection in our analysis [10,21]. For classical epidemic models, it is common that the basic reproduction number is a threshold in the sense that a disease will survive if the basic reproductive number is greater than one, and it will die out if it is less than one. In some cases, the basic reproduction number may not reflect the elimination effort required; instead, the value of the critical parameter represents the effort at the turning point. This phenomenon is known as backward bifurcation. Thus, it is important to examine the occurrence of backward bifurcations to achieve thresholds for disease control. The presence of a backward bifurcation and the circumstances for its appearance in epidemic models have been extensively studied over the years. The problem of determining the causes of backward bifurcation in some standard deterministic models for the spread of some emerging and re-emerging diseases were studied by Gumel [13]. Recently, the necessary and sufficient conditions for the occurrence of backward bifurcation in general epidemic models have been studied by [23]. Backward bifurcation has been studied for several epidemic diseases [11,24,32]. However, we have studied this phenomenon here for our proposed models of MERS-CoV transmission. Our objective is not only to investigate the qualitative effect of disease transmission but also to effectively control the disease as quickly and efficiently as possible. Improving control and finally eradicating MERS-CoV from the population is one of the key reasons for investigating this infectious disease. There are various types of control mechanisms that have been studied in literature over the years. In the literature, several nonlinear control methods are also suggested. The rapid growth of Networked control systems has been followed by some thorny issues, such as network delay, imperfect communication links and limited communication bandwidth [31]. They studied the state estimation problem for the set of switched complex dynamic networks affected by the quantization, in which the switching mechanism is presumed to obey persistent dwell-time switching regulations. Many researchers have studied to unify a variety of different performance indexes within a uniform framework. More general performance called extended dissipative performance, which addressed four common performance indexes in a detailed manner within 
Fig. 1 Compartmental flow diagram of the proposed model. Here green color indicates the susceptible compartment and the red color is for the infected compartment. The dotted double arrows denote the contacts between susceptible and infected populations and single sided blue solid arrows represent transition from one compartment to another. Here the green dotted line represents the contact within the human population and the red dotted line represents the contact between the human and camel population

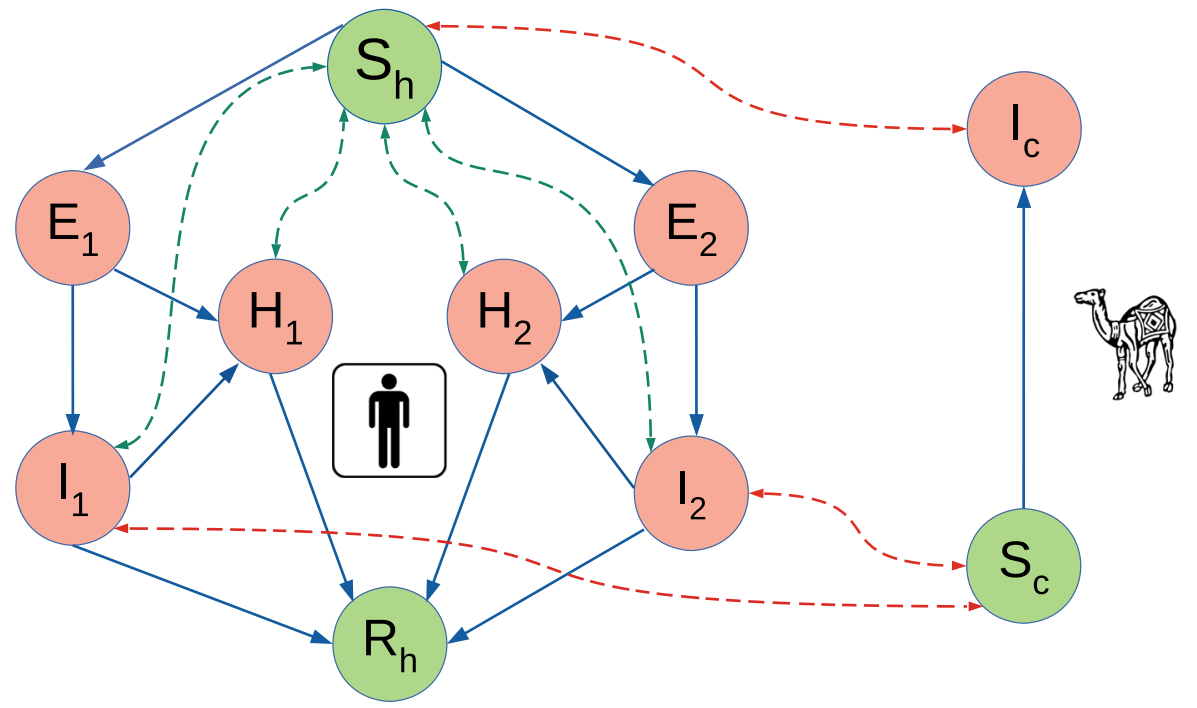

the same framework [37]. Extended dissipation was used in several systems analysis. The analyses of the extended dissipative performance of the closed-loop PDT SPSSs and a preferable decoupling method deriving the mode-dependent controller gains are given in [30]. Time-dependent control strategies may be applied over a finite time interval to prevent a disease. Optimal control is a powerful mathematical method that can be used in this situation to make decisions [20]. Here, we conduct optimal control analysis on various combined interventions with four components, namely preventive measures such as mask use, isolation of strain-1 infected individuals, strain-2 infected individuals and infected camels.

The rest of the paper is organized as follows: in Sect. 2, we formulate the compartmental model of MERS-CoV; the single strain version of the proposed model is analyzed in Sect. 3; optimal control analysis and numerical simulations for the proposed two strain model is presented in Sect. 4; finally, the obtained results are discussed for prevention strategies in Sect. 5.

\section{Model formulation}

We consider two heterogeneous population groups as host and vector population. Time-dependent state variables are taken to describe the compartments of the two populations. Let at time t, $N_{h}(t)$ represents total host population and $N_{c}(t)$ represents total camel population as vector. We consider an extension of 'SEIR' type
Table 1 Description of variables used in the model

\begin{tabular}{ll}
\hline Variables & Interpretation \\
\hline$S_{h}$ & Susceptible human population \\
$E_{i}$ & $\begin{array}{l}\text { Exposed human population with strain } i, \\
i=1,2\end{array}$ \\
& Un-notified infected human population \\
$I_{i}$ & with strain $i, i=1,2$ \\
& Hospitalized and/or notified infected \\
$H_{i}$ & population with strain $i, i=1,2$ \\
& Recovered human population \\
$R_{h}$ & Susceptible camel population \\
$S_{c}$ & Infected camel population \\
$I_{c}$ & \\
\hline
\end{tabular}

model for the host population and a 'SI' type model for the vectors. We subdivide the host population into eight mutually disjoint classes: susceptible, $S_{h}$; Exposed with strain-i, $E_{i}$; un-notified infected with strain-i, $I_{i}$; Hospitalized or notified with strain-i, $H_{i}$; recovered, $R_{h}$ $(i=1,2)$. Thus, at any time t, the size of the human population is given by $N_{h}=S_{h}+\sum_{i=1}^{2}\left(E_{i}+I_{i}+A_{i}\right)+R_{h}$. The vector population is divided into two classes such that at time $t$, there are susceptible, $S_{c}$; and infected, $I_{c}$ vectors. With this division, the size of the vector population at time $t$ is given by $N_{c}(t)=S_{c}(t)+I_{c}(t)$. A flow diagram of the proposed model is depicted in Fig. 1.

We assume all newborn hosts are fully susceptible. The susceptible host population increases at a constant rate $\Pi_{h}$. The susceptible population decreases due to 
Table 2 Description of parameters used in the model

\begin{tabular}{|c|c|c|c|}
\hline Parameters & Interpretation & Value & Reference \\
\hline$\Pi_{h}$ & Recruitment rate of host & 1000 week $^{-1}$ & - \\
\hline$\Pi_{c}$ & Recruitment rate of camel & 50 week $^{-1}$ & - \\
\hline$\mu_{h}$ & Natural death rate of host & $2.54 \times 10^{-4}$ week $^{-1}$ & {$[35]$} \\
\hline$\mu_{c}$ & Natural death rate of camel & 0.00048 week $^{-1}$ & {$[34]$} \\
\hline$\beta_{1}$ & Transmission rate from strain- 1 infected humans to susceptible humans & 0.75 & - \\
\hline$\beta_{2}$ & Transmission rate from strain-2 infected humans to susceptible humans & 0.56 & - \\
\hline$\beta_{3}$ & Transmission rate from strain-1 infected humans to susceptible camels & 0.7 & - \\
\hline$\beta_{4}$ & Transmission rate from strain-2 infected humans to susceptible camels & 0.3 & - \\
\hline$\beta_{d}$ & Transmission rate from infected camel to susceptible humans & 0.75 & - \\
\hline$\alpha_{i}$ & Modification parameters & 2 & {$[6]$} \\
\hline$m$ & Modification parameter & 0.5 & - \\
\hline $1 / \gamma_{i}$ & Incubation period & 0.7429 week & {$[2,38]$} \\
\hline$r_{1}$ & Proportion of strain-1 infected people who are un-notified & 0.6 & - \\
\hline$r_{2}$ & Proportion of strain-2 infected people who are un-notified & 0.8 & - \\
\hline$k_{1 i}$ & Recovery rate of un-notified infected human class & 0.6 week $^{-1}$ & - \\
\hline$k_{2 i}$ & Recovery rate of hospitalized human class & 0.7 week $^{-1}$ & - \\
\hline$\eta_{i}$ & Transmission rate from infected humans to hospitalized humans & 0.001 week $^{-1}$ & - \\
\hline$\delta_{i}$ & Disease induced mortality rate & $2.99 \times 10^{-8}$ & {$[9,33]$} \\
\hline
\end{tabular}

getting infection from infected vectors and infected hosts and natural mortality at a rate $\mu_{h}$, hence the average life span of the human population in endemic region is $\frac{1}{\mu_{h}}$. After getting infection the susceptible hosts progress to either $E_{1}$ or $E_{2}$. From $E_{1}$, people may progress to either $I_{1}$ or $H_{1}$ after incubation period is over. Similar progression is assumed for strain-2. The infected human populations $I_{i}$ and $H_{i}$ decreases as a result of natural death and recovery. We also consider disease induced mortality in the hospitalized compartment as MERS-CoV has a high case fatality rate. The average life span of vector population is $\frac{1}{\mu_{c}}$, that means $\mu_{c}$ is the natural mortality rate of camels. The susceptible camel population increases at a constant rate $\Pi_{c}$. All the parameters used in the system are described in Table 2. The above assumptions lead to the following set of ordinary differential equations:

$$
\begin{aligned}
\frac{\mathrm{d} S_{h}}{\mathrm{~d} t}= & \Pi_{h}-\beta_{1} \frac{S_{h}}{N_{h}}\left(I_{1}+\alpha_{1} H_{1}\right) \\
& -\beta_{2} \frac{S_{h}}{N_{h}}\left(I_{2}+\alpha_{2} H_{2}\right)-\beta_{d} \frac{S_{h}}{N_{h}} I_{c}-\mu_{h} S_{h}, \\
\frac{\mathrm{d} E_{1}}{\mathrm{~d} t}= & \beta_{1} \frac{S_{h}}{N_{h}}\left(I_{1}+\alpha_{1} H_{1}\right)
\end{aligned}
$$

$$
\begin{aligned}
& +m \beta_{d} \frac{S_{h}}{N_{h}} I_{c}-\mu_{h} E_{1}-\gamma_{1} E_{1}, \\
\frac{\mathrm{d} E_{2}}{\mathrm{~d} t}= & \beta_{2} \frac{S_{h}}{N_{h}}\left(I_{2}+\alpha_{2} H_{2}\right) \\
& +(1-m) \beta_{d} \frac{S_{h}}{N_{h}} I_{c}-\mu_{h} E_{2}-\gamma_{2} E_{2}, \\
\frac{\mathrm{d} I_{1}}{\mathrm{~d} t}= & r_{1} \gamma_{1} E_{1}-\left(\mu_{h}+k_{11}+\eta_{1}\right) I_{1}, \\
\frac{\mathrm{d} I_{2}}{\mathrm{~d} t}= & r_{2} \gamma_{2} E_{2}-\left(\mu_{h}+k_{12}+\eta_{2}\right) I_{2}, \\
\frac{\mathrm{d} H_{1}}{\mathrm{~d} t}= & \left(1-r_{1}\right) \gamma_{1} E_{1}+\eta_{1} I_{1}-\left(\mu_{h}+k_{21}+\delta_{1}\right) H_{1}, \\
\frac{\mathrm{d} H_{2}}{\mathrm{~d} t}= & \left(1-r_{2}\right) \gamma_{2} E_{2}+\eta_{2} I_{2}-\left(\mu_{h}+k_{22}+\delta_{2}\right) H_{2}, \\
\frac{\mathrm{d} R_{h}}{\mathrm{~d} t}= & k_{11} I_{1}+k_{12} I_{2}+k_{21} H_{1}+k_{22} H_{2}-\mu_{h} R_{h}, \\
\frac{\mathrm{d} S_{c}}{\mathrm{~d} t}= & \Pi_{c}-\beta_{3} \frac{S_{c} I_{1}}{N_{h}}-\beta_{4} \frac{S_{c} I_{2}}{N_{h}}-\mu_{c} S_{c}, \\
\frac{\mathrm{d} I_{c}}{\mathrm{~d} t}= & \beta_{3} \frac{S_{c} I_{1}}{N_{h}}+\beta_{4} \frac{S_{c} I_{2}}{N_{h}}-\mu_{c} I_{c} .
\end{aligned}
$$

The variables and all the parameters and their biological interpretation are given in Tables 1 and 2, respectively. 


\section{Model analysis for single strain}

In this section, we analyze the single starin version of model 1 mathematically. Here we consider the single strain model as follows:

$\frac{\mathrm{d} S_{h}}{\mathrm{~d} t}=\Pi_{h}-\beta_{1} \frac{S_{h}}{N_{h}}\left(I_{1}+\alpha_{1} H_{1}\right)-\beta_{d} \frac{S_{h}}{N_{h}} I_{c}-\mu_{h} S_{h}$

$\frac{\mathrm{d} E_{1}}{\mathrm{~d} t}=\beta_{1} \frac{S_{h}}{N_{h}}\left(I_{1}+\alpha_{1} H_{1}\right)+\beta_{d} \frac{S_{h}}{N_{h}} I_{c}-\mu_{h} E_{1}-\gamma_{1} E_{1}, \quad \frac{\mathrm{d} X}{\mathrm{~d} t}=M X+N$

$\frac{\mathrm{d} I_{1}}{\mathrm{~d} t}=r_{1} \gamma_{1} E_{1}-\left(\mu_{h}+k_{11}+\eta_{1}\right) I_{1}$, $\frac{\mathrm{d} N_{c}}{\mathrm{~d} t}=\Pi_{c}-\mu_{c} N_{c}$
$\Longrightarrow \lim _{t \rightarrow \infty} \sup _{c}(t) \rightarrow \frac{\Pi_{c}}{\mu_{c}}$

Now the system (2) can be written as

where $X=\left(S_{h}, E_{1}, I_{1}, H_{1}, R_{h}, S_{c}, I_{c}\right)^{T}$. The matrix $M$ is given by

$$
M=\left(\begin{array}{ccccccc}
-\left(\theta_{1}+\theta_{2}+\mu_{h}\right) & 0 & 0 & 0 & 0 & 0 & 0 \\
\theta_{1}+\theta_{2} & -\left(\mu_{h}+\gamma_{1}\right) & 0 & 0 & 0 & 0 & 0 \\
0 & r_{1} \gamma_{1} & -\left(\mu_{h}+k_{11}+\eta_{1}\right) & 0 & 0 & 0 & 0 \\
0 & \left(1-r_{1}\right) \gamma_{1} & \eta_{1} & -\left(\mu_{h}+k_{21}+\delta_{1}\right) & 0 & 0 & 0 \\
0 & 0 & k_{11} & k_{21} & -\mu_{h} & 0 & 0 \\
0 & 0 & 0 & 0 & 0 & -\left(\theta_{3}+\mu_{c}\right) & 0 \\
0 & 0 & 0 & 0 & 0 & \theta_{3} & -\mu_{c}
\end{array}\right)
$$

$\frac{\mathrm{d} H_{1}}{\mathrm{~d} t}=\left(1-r_{1}\right) \gamma_{1} E_{1}+\eta_{1} I_{1}-\left(\mu_{h}+k_{21}+\delta_{1}\right) H_{1}$,

$\frac{\mathrm{d} R_{h}}{\mathrm{~d} t}=k_{11} I_{1}+k_{21} H_{1}-\mu_{h} R_{h}$,

$\frac{\mathrm{d} S_{c}}{\mathrm{~d} t}=\Pi_{c}-\beta_{3} \frac{S_{c} I_{1}}{N_{h}}-\mu_{c} S_{c}$,

$\frac{\mathrm{d} I_{c}}{\mathrm{~d} t}=\beta_{3} \frac{S_{c} I_{1}}{N_{h}}-\mu_{c} I_{c}$.

\subsection{Mathematical analysis}

\subsubsection{Positivity and boundedness of the solution}

Proposition 1 The system (2) is bounded in the region $\Omega=\left\{\left(S_{h}, E_{1}, I_{1}, H_{1}, R_{h}, S_{c}, I_{c}\right) \in R_{+}^{7} \mid S_{h}+E_{1}+\right.$ $\left.I_{1}+H_{1}+R_{h} \leq \frac{\Pi_{h}}{\mu_{h}}, S_{c}+I_{c} \leq \frac{\Pi_{c}}{\mu_{c}}\right\}$, which is positively invariant.

Proof We observed from the system that

$$
\begin{aligned}
& \frac{\mathrm{d} N_{h}}{\mathrm{~d} t}=\Pi_{h}-\mu_{h} N_{h}-\delta_{1} H_{1} \leq \Pi_{h}-\mu_{h} N_{h} \\
& \Longrightarrow \lim _{t \rightarrow \infty} \sup _{h}(t) \rightarrow \frac{\Pi_{h}}{\mu_{h}}
\end{aligned}
$$

and where

$\theta_{1}=\frac{\beta_{1}\left(I_{1}+\alpha_{1} H_{1}\right)}{N_{h}}, \theta_{2}=\frac{\beta_{d} I_{c}}{N_{h}}, \theta_{3}=\frac{\beta_{3} I_{1}}{N_{h}}$

The vector $N=\left(\Pi_{h}, 0,0,0,0,0, \Pi_{c}, 0\right)^{T}$. Note that $M X$ is a Metzler matrix for all $X \in R_{+}^{7}$. Since $N \geq 0$, system (2) is positively invariant in $R_{+}^{7}$ [1]. Therefore, every trajectory of the system (2) starting from an initial state in $R_{+}^{7}$ remains trapped in $R_{+}^{7}$.

Therefore, all mathematically and biologically feasible solutions of the model system (2) are attractive to the region $\Omega$. Of this reason, it is now sufficient to analyze the dynamic properties of the Model (2) in $\Omega$.

\subsubsection{Diseases-free equilibrium and basic reproduction number}

The diseases-free equilibrium can be obtained for the system (2) by putting $E_{1}=0, I_{1}=0, H_{1}=0, I_{c}=$ 0 , which is denoted by $P_{0}=\left(S_{h}, 0,0,0, R_{h}, S_{c}, 0\right)$, where

$$
S_{h}=\frac{\Pi_{h}}{\mu_{h}}, S_{c}=\frac{\Pi_{c}}{\mu_{c}}, R_{h}=0 .
$$


The basic reproduction number, a key idea in the study of the spread of infectious diseases, is the threshold value that specifies the number of infected individuals generated by one infected individual in a completely susceptible population. It is denoted by $R_{01}$ and calculated as $R_{d}+R_{v}$, where $R_{d}$ is the direct transmission reproduction number and $R_{v}$ is the vector transmission reproduction number [3]. This dimensionless number is determined by the next generation operator method at DFE. For this reason, we assemble the compartments

$$
\begin{aligned}
F & =\frac{\partial \mathcal{F}}{\partial X}=\left(\begin{array}{cccc}
0 & \beta_{1} & \alpha_{1} \beta_{1} & \beta_{d} \\
0 & 0 & 0 & 0 \\
0 & 0 & 0 & 0 \\
0 & \beta_{3} \frac{\Pi_{c} \mu_{h}}{\Pi_{h} \mu_{c}} & 0 & 0
\end{array}\right), \\
V & =\frac{\partial \mathcal{V}}{\partial X}=\left(\begin{array}{cccc}
\mu_{h}+\gamma_{1} & 0 & 0 & 0 \\
-r_{1} \gamma_{1} & \mu_{h}+k_{11}+\eta_{1} & 0 & 0 \\
-\left(1-r_{1}\right) \gamma_{1} & -\eta_{1} & \mu_{h}+k_{21}+\delta_{1} & 0 \\
0 & 0 & 0 & \mu_{c}
\end{array}\right) .
\end{aligned}
$$

Now the $V^{-1}$ is given by

$V^{-1}=\left(\begin{array}{cccc}\frac{1}{\mu_{h}+\gamma_{1}} & 0 & 0 & 0 \\ \frac{r_{1} \gamma_{1}}{\left(\mu_{h}+\gamma_{1}\right)\left(\mu_{h}+k_{11}+\eta_{1}\right)} & \frac{1}{\mu_{h}+k_{11}+\eta_{1}} & 0 & 0 \\ \frac{\eta_{1} \gamma_{1} \eta_{1}+\left(1-r_{1}\right) \gamma_{1}\left(\mu_{h}+k_{11}+\eta_{1}\right)}{\left(\mu_{h}+\gamma_{1}\right)\left(\mu_{h}+k_{11}+\eta_{1}\right)\left(\mu_{h}+k_{21}+\delta_{1}\right)} & \frac{1}{\left(\mu_{h}+k_{11}+\eta_{1}\right)\left(\mu_{h}+k_{21}+\delta_{1}\right)} & \frac{1}{\mu_{h}+k_{21}+\delta_{1}} & 0 \\ 0 & 0 & 0 & \frac{1}{\mu_{c}}\end{array}\right)$

Thus, the matrix $F V^{-1}$ is given as

$F V^{-1}=\left(\begin{array}{cccc}\frac{\beta_{1} r_{1} \gamma_{1}}{m_{1} m_{2}}+\frac{\alpha_{1} \beta_{1}\left[r_{1} \gamma_{1} \eta_{1}+\left(1-r_{1}\right) \gamma_{1} m_{2}\right]}{m_{1} m_{2} m_{3}} & \frac{\beta_{1}}{m_{2}}+\frac{\alpha_{1} \beta_{1} \eta_{1}}{m_{2} m_{3}} & \frac{\alpha_{1} \beta_{1}}{m_{3}} & \frac{\beta_{d}}{\mu_{c}} \\ 0 & 0 & 0 & 0 \\ 0 & 0 & 0 & 0 \\ \beta_{3} \frac{\Pi_{c} \mu_{h}}{\Pi_{h} \mu_{c}} \frac{r_{1} \gamma_{1}}{m_{1} m_{2}} & \beta_{3} \frac{\Pi_{c} \mu_{h}}{\Pi_{h} \mu_{c}} \frac{1}{m_{2}} & 0 & 0\end{array}\right)$,

that are infected from the system (2) and decompose the right side as $\mathcal{F}-\mathcal{V}$, where $\mathcal{F}$ is the transmission part, which expresses the generation of new infections, and the transition part is $\mathcal{V}$, which describes the change in state.

$$
\begin{aligned}
& \mathcal{F}=\left(\begin{array}{c}
\beta_{1} \frac{S_{h}}{N_{h}}\left(I_{1}+\alpha_{1} H_{1}\right)+\beta_{d} \frac{S_{h}}{N_{h}} I_{c} \\
0 \\
0 \\
\beta_{3} \frac{S_{c}}{N_{h}} I_{h}
\end{array}\right), \\
& \mathcal{V}=\left(\begin{array}{c}
\mu_{h} E_{1}+\gamma_{1} E_{1} \\
-r_{1} \gamma_{1} E_{1}+\left(\mu_{h}+k_{11}+\eta_{1}\right) \\
-\left(1-r_{1}\right) \gamma_{1} E_{1}-\eta_{1} I_{1}+\left(\mu_{h}+k_{21}+\delta_{1}\right) H_{1} \\
\mu_{c} I_{c}
\end{array}\right)
\end{aligned}
$$

where $m_{1}=\mu_{h}+\gamma_{1}, m_{2}=\mu_{h}+k_{11}+\eta_{1}$ and $m_{3}=$ $\mu_{h}+k_{21}+\delta_{1}$.

To find $R_{d}$ we use $\beta_{d}=0$ and $\beta_{3}=0$ in $F$, then the direct transmission reproduction number $R_{d}$ for the Model (2) is given by

$$
\begin{aligned}
F_{1} & =\left(\begin{array}{cccc}
0 & \beta_{1} & \alpha_{1} \beta_{1} & 0 \\
0 & 0 & 0 & 0 \\
0 & 0 & 0 & 0 \\
0 & 0 & 0 & 0
\end{array}\right), \\
V & =\left(\begin{array}{cccc}
\mu_{h}+\gamma_{1} & 0 & 0 & 0 \\
-r_{1} \gamma_{1} & \mu_{h}+k_{11}+\eta_{1} & 0 & 0 \\
-\left(1-r_{1}\right) \gamma_{1} & -\eta_{1} & \mu_{h}+k_{21}+\delta_{1} & 0 \\
0 & 0 & 0 & \mu_{c}
\end{array}\right) .
\end{aligned}
$$

Now the direct transmission reproduction number $R_{d}$ is the spectral radius of $F_{1} V^{-1}$, therefore

Now we calculate the Jacobian of $\mathcal{F}$ and $\mathcal{V}$ at DFE $P_{0}$ 


$$
\begin{aligned}
R_{d}= & \frac{\beta_{1} r_{1} \gamma_{1}}{\left(\mu_{h}+\gamma_{1}\right)\left(\mu_{h}+k_{11}+\eta_{1}\right)} \\
& +\frac{\alpha_{1} \beta_{1} r_{1} \gamma_{1} \eta_{1}}{\left(\mu_{h}+\gamma_{1}\right)\left(\mu_{h}+k_{11}+\eta_{1}\right)\left(\mu_{h}+k_{21}+\delta_{1}\right)} \\
& +\frac{\alpha_{1} \beta_{1}\left(1-r_{1}\right) \gamma_{1}}{\left(\mu_{h}+\gamma_{1}\right)\left(\mu_{h}+k_{21}+\delta_{1}\right)}
\end{aligned}
$$

To find $R_{v}$ we use $\beta_{h}=0$ in $F$. Therefore, the vector transmission reproduction number $R_{v}$ is given by

$$
\begin{aligned}
F_{2} & =\left(\begin{array}{cccc}
0 & 0 & 0 & \beta_{d} \\
0 & 0 & 0 & 0 \\
0 & 0 & 0 & 0 \\
0 & \beta_{c} \frac{\Pi_{c} \mu_{h}}{\Pi_{h} \mu_{c}} & 0 & 0
\end{array}\right), \\
V & =\left(\begin{array}{cccc}
\mu_{h}+\gamma_{1} & 0 & 0 & 0 \\
-r_{1} \gamma_{1} & \mu_{h}+k_{11}+\eta_{1} & 0 & 0 \\
-\left(1-r_{1}\right) \gamma_{1} & -\eta_{1} & \mu_{h}+k_{21}+\delta_{1} & 0 \\
0 & 0 & 0 & \mu_{c}
\end{array}\right) .
\end{aligned}
$$

Therefore, the basic reproduction number is

$$
\begin{aligned}
R_{01} & =R_{d}+R_{v} \\
& =\frac{\beta_{1} r_{1} \gamma_{1}}{\left(\mu_{h}+\gamma_{1}\right)\left(\mu_{h}+k_{11}+\eta_{1}\right)} \\
& +\frac{\alpha_{1} \beta_{1} r_{1} \gamma_{1} \eta_{1}}{\left(\mu_{h}+\gamma_{1}\right)\left(\mu_{h}+k_{11}+\eta_{1}\right)\left(\mu_{h}+k_{21}+\delta_{1}\right)} \\
& +\frac{\alpha_{1} \beta_{1}\left(1-r_{1}\right) \gamma_{1}}{\left(\mu_{h}+\gamma_{1}\right)\left(\mu_{h}+k_{21}+\delta_{1}\right)} \\
& +\frac{\beta_{3} \beta_{d} r_{1} \gamma_{1} \Pi_{c} \mu_{h}}{\mu_{c}^{2} \Pi_{h}\left(\mu_{h}+\gamma_{1}\right)\left(\mu_{h}+k_{11}+\eta_{1}\right)}
\end{aligned}
$$

\subsubsection{Stability of DFE}

Theorem 1 The diseases free equilibrium $(D F E) P_{0}=$ $\left(\frac{\Pi_{h}}{\mu_{h}}, 0,0,0,0, \frac{\Pi_{c}}{\mu_{c}}, 0\right)$ of the system (2) is locally asymptotically stable if $R_{01}<1$ and unstable if $R_{01}>1$.

Proof We calculate the Jacobian of the system (2) at DFE, and is given by

$$
J_{P_{0}}=\left(\begin{array}{ccccccc}
-\mu_{h} & 0 & -\beta_{1} & -\alpha_{1} \beta_{1} & 0 & 0 & -\beta_{d} \\
0 & -\left(\mu_{h}+\gamma_{1}\right) & \beta_{1} & \alpha_{1} \beta_{1} & 0 & 0 & \beta_{d} \\
0 & r_{1} \gamma_{1} & -\left(\mu_{h}+k_{11}+\eta_{1}\right) & 0 & 0 & 0 & 0 \\
0 & \left(1-r_{1}\right) \gamma_{1} & \eta_{1} & -\left(\mu_{h}+k_{21}+\delta_{1}\right) & 0 & 0 & 0 \\
0 & 0 & k_{11} & k_{21} & -\mu_{h} & 0 & 0 \\
0 & 0 & -\beta_{3} \frac{\Pi_{c} \mu_{h}}{\Pi_{h} \mu_{c}} & 0 & 0 & -\mu_{c} & 0 \\
0 & 0 & \beta_{3} \frac{\Pi_{c} \mu_{h}}{\Pi_{h} \mu_{c}} & 0 & 0 & 0 & -\mu_{c}
\end{array}\right),
$$

Now the vector transmission reproduction number $R_{v}$ is the spectral radius of $F_{2} V^{-1}$, therefore

$$
R_{v}=\sqrt{\frac{\beta_{3} \beta_{d} r_{1} \gamma_{1} \Pi_{c} \mu_{h}}{\mu_{c}^{2} \Pi_{h}\left(\mu_{h}+\gamma_{1}\right)\left(\mu_{h}+k_{11}+\eta_{1}\right)}}
$$

In this calculation, the transition is considered as two generations. In studying vector transmitted diseases it is common to consider this as one generations and use the value by direct approach

$$
R_{v}=\frac{\beta_{3} \beta_{d} r_{1} \gamma_{1} \Pi_{c} \mu_{h}}{\mu_{c}^{2} \Pi_{h}\left(\mu_{h}+\gamma_{1}\right)\left(\mu_{h}+k_{11}+\eta_{1}\right)}
$$

Let $\lambda$ be the eigenvalue of the matrix $J_{P_{0}}$. Then the characteristic equation is given by $\operatorname{det}\left(J_{P_{0}}-\lambda I\right)=0$. which implies

$$
\begin{gathered}
\beta_{1} r_{1} \gamma_{1} \mu_{c}\left(\mu_{h}+k_{21}+\delta_{1}+\lambda\right)+\alpha_{1} \beta_{1} r_{1} \gamma_{1} \mu_{c} \\
+\alpha_{1} \beta_{1} \gamma_{1} \mu_{c}\left(1-r_{1}\right)\left(\mu_{h}+k_{11}+\eta_{1}+\lambda\right) \\
+\beta_{d} \beta_{c} r_{a} \gamma_{1}\left(\mu_{h}+k_{21}+\delta_{1}+\lambda\right) \frac{\Pi_{c} \mu_{h}}{\Pi_{h} \mu_{c}} \\
-\left(\mu_{h}+\gamma_{1}+\lambda\right)\left(\mu_{h}+k_{11}+\eta_{1}+\lambda\right) \\
\quad\left(\mu_{h}+k_{21}+\delta_{1}+\lambda\right)=0
\end{gathered}
$$


Which can be written as

$$
\begin{aligned}
& \frac{\beta_{1} r_{1} \gamma_{1}}{\left(\mu_{h}+\gamma_{1}+\lambda\right)\left(\mu_{h}+k_{11}+\eta_{1}+\lambda\right)} \\
& +\frac{\alpha_{1} \beta_{1} r_{1} \gamma_{1} \eta_{1}}{\left(\mu_{h}+\gamma_{1}+\lambda\right)\left(\mu_{h}+k_{11}+\eta_{1}+\lambda\right)\left(\mu_{h}+k_{21}+\delta_{1}+\lambda\right)} \\
& +\frac{\alpha_{1} \beta_{1}\left(1-r_{1}\right) \gamma_{1}}{\left(\mu_{h}+\gamma_{1}+\lambda\right)\left(\mu_{h}+k_{21}+\delta_{1}+\lambda\right)} \\
& +\frac{\beta_{3} \beta_{d} r_{1} \gamma_{1} \Pi_{c} \mu_{h}}{\mu_{c}^{2} \Pi_{h}\left(\mu_{h}+\gamma_{1}+\lambda\right)\left(\mu_{h}+k_{11}+\eta_{1}+\lambda\right)}=1 .
\end{aligned}
$$

Denote

$$
\begin{aligned}
G(\lambda)= & \frac{\beta_{1} r_{1} \gamma_{1}}{\left(\mu_{h}+\gamma_{1}+\lambda\right)\left(\mu_{h}+k_{11}+\eta_{1}+\lambda\right)} \\
& +\frac{\alpha_{1} \beta_{1} r_{1} \gamma_{1} \eta_{1}}{\left(\mu_{h}+\gamma_{1}+\lambda\right)\left(\mu_{h}+k_{11}+\eta_{1}+\lambda\right)\left(\mu_{h}+k_{21}+\delta_{1}+\lambda\right)} \\
& +\frac{\alpha_{1} \beta_{1}\left(1-r_{1}\right) \gamma_{1}}{\left(\mu_{h}+\gamma_{1}+\lambda\right)\left(\mu_{h}+k_{21}+\delta_{1}+\lambda\right)} \\
& +\frac{\beta_{3} \beta_{d} r_{1} \gamma_{1} \Pi_{c} \mu_{h}}{\mu_{c}^{2} \Pi_{h}\left(\mu_{h}+\gamma_{1}+\lambda\right)\left(\mu_{h}+k_{11}+\eta_{1}+\lambda\right)} .
\end{aligned}
$$

We rewrite $G(\lambda)$ as $G(\lambda)=G_{1}(\lambda)+G_{2}(\lambda)+G_{3}(\lambda)+$ $G_{4}(\lambda)$

Now if $\operatorname{Re}(\lambda) \geq 0, \lambda=x+i y$, then

$$
\begin{aligned}
\left|G_{1}(\lambda)\right| & \leq \frac{\beta_{1} r_{1} \gamma_{1}}{\left|\mu_{h}+\gamma_{1}+\lambda\right|\left|\mu_{h}+k_{11}+\eta_{1}+\lambda\right|} \\
& \leq G_{1}(x) \leq G_{1}(0) \\
\left|G_{2}(\lambda)\right| & \leq \frac{\alpha_{1} \beta_{1} r_{1} \gamma_{1} \eta_{1}}{\left|\mu_{h}+\gamma_{1}+\lambda\right|\left|\mu_{h}+k_{11}+\eta_{1}+\lambda\right|\left|\mu_{h}+k_{21}+\delta_{1}+\lambda\right|} \\
& \leq G_{2}(x) \leq G_{2}(0) \\
\left|G_{3}(\lambda)\right| & \leq \frac{\alpha_{1} \beta_{1}\left(1-r_{1}\right) \gamma_{1}}{\left|\mu_{h}+\gamma_{1}+\lambda\right|\left|\mu_{h}+k_{21}+\delta_{1}+\lambda\right|} \\
& \leq G_{3}(x) \leq G_{3}(0) \\
\left|G_{4}(\lambda)\right| & \leq \frac{\beta_{3} \beta_{d} r_{1} \gamma_{1} \Pi_{c} \mu_{h}}{\mu_{c}^{2} \Pi_{h}\left|\mu_{h}+\gamma_{1}+\lambda\right|\left|\mu_{h}+k_{11}+\eta_{1}+\lambda\right|} \\
& \leq G_{4}(x) \leq G_{4}(0)
\end{aligned}
$$

Then $G_{1}(0)+G_{2}(0)+G_{3}(0)+G_{4}(0)=G(0)=$ $R_{01}<1$, which implies $|G(\lambda)| \leq 1$.

Thus for $R_{01}<1$, all the eigenvalues of the characteristics equation $G(\lambda)=1$ has negative real parts.

Therefore, if $R_{01}<1$, all eigenvalues are negative and hence DFE $P^{0}$ is locally asymptotically stable.

Now if we consider $R_{01}>1$ i.e $G(0)>1$, then

$\lim _{\lambda \rightarrow \infty} G(\lambda)=0$.

Then there exist $\lambda_{1}^{*}>0$ such that $G\left(\lambda_{1}^{*}\right)=1$.

That means there exists positive eigenvalue $\lambda_{1}^{*}>0$ of the Jacobian matrix.
Hence, DFE $P_{0}$ is unstable whenever $R_{01}>1$.

\subsubsection{Global stability of DFE:}

Now set $X_{1}=\left(S_{h}, R_{h}, S_{c}\right)$ and $X_{2}=\left(I_{1}, H_{1}, I_{1}, E_{1}\right)$. Then the system can be written on the set $\Omega$, in a pseudo-triangular form

$\dot{X}_{1}=A_{1}(X)\left(X_{1}-X_{1}^{*}\right)+A_{12}(X) X_{2}$

$\dot{X}_{2}=A_{2}(X) X_{2}$

We express the sub-system $\dot{X}_{1}=A_{1}(X)\left(X_{1}-X_{1}^{*}\right)+$ $A_{12}(X) X_{2}$

$$
\begin{aligned}
\dot{S}_{h} & =\Pi_{h}-\mu_{h} S_{h} \\
\dot{R}_{h} & =-\mu_{h} R_{h} \\
\dot{S}_{c} & =\Pi_{c}-\mu_{c} S_{c}
\end{aligned}
$$

This is a linear system which is globally asymptotically stable at the equilibrium $\left(\frac{\Pi_{h}}{\mu_{h}}, 0, \frac{\Pi_{c}}{\mu_{c}}\right)$ corresponding to the DFE which satisfies $\mathrm{H} 1$ and $\mathrm{H} 2$ [17].

$$
\begin{aligned}
& \text { The matrix } A_{2}(X) \text { is given by } A_{2}(X)= \\
& \left(\begin{array}{cccc}
-\mu_{c} & 0 & \beta_{3} \frac{S_{c}}{N_{h}} & 0 \\
0 & -\left(\mu_{h}+k_{21}+\delta_{1}\right) & \eta_{1} & \left(1-r_{1}\right) \gamma_{1} \\
0 & 0 & -\left(\mu_{h}+k_{11}+\eta_{1}\right) & r_{1} \gamma_{1} \\
\beta_{d} \frac{S_{h}}{N_{h}} & \alpha_{1} \beta_{h} \frac{S_{h}}{N_{h}} & \beta_{h} \frac{S_{h}}{N_{h}} & -\left(\mu_{h}+\gamma_{1}\right)
\end{array}\right)
\end{aligned}
$$

which is an irreducible matrix.

The upper bound for $X \in \Omega$ is given by $\overline{A_{2}}=$ $\left(\begin{array}{cccc}-\mu_{c} & 0 & \beta_{3} \frac{\Pi_{c}\left(\mu_{h}+\delta_{1}\right)}{\Pi_{h} \mu_{c}} & 0 \\ 0 & -\left(\mu_{h}+k_{21}+\delta_{1}\right) & \eta_{1} & \left(1-r_{1}\right) \gamma_{1} \\ 0 & 0 & -\left(\mu_{h}+k_{11}+\eta_{1}\right) & r_{1} \gamma_{1} \\ \beta_{d} & \alpha_{1} \beta_{h} & \beta_{h} & -\left(\mu_{h}+\gamma_{1}\right)\end{array}\right)$ which is not attained on $\Omega$ and which is not the corresponding block in Jacobian matrix of the system at the DFE. Thus we obtain only sufficient condition.

It is straightforward to check that all hypothesis of Theorem 4.3 of [17] are satisfied. Hence, we obtained the condition

$$
R_{01}+\frac{\beta_{3} \beta_{d} r_{1} \gamma_{1} \Pi_{c} \delta_{1}}{\mu_{c}^{2} \Pi_{h}\left(\mu_{h}+\gamma_{1}\right)\left(\mu_{h}+k_{11}+\eta_{1}\right)}<1
$$

Which implies

$$
R_{01}<1-\frac{\beta_{3} \beta_{d} r_{1} \gamma_{1} \Pi_{c} \delta_{1}}{\mu_{c}^{2} \Pi_{h}\left(\mu_{h}+\gamma_{1}\right)\left(\mu_{h}+k_{11}+\eta_{1}\right)}=R_{01}^{c} \leq 1
$$

Obviously $R_{01}<R_{01}^{c}$ and this condition is stronger. 
Theorem 2 The diseases free equilibrium (DFE) $P_{0}=$ $\left(S_{h}, 0,0,0, R_{h}, S_{c}, 0\right)$ of the system (1) is globally stable if $R_{01}<R_{01}^{c}$.

\subsubsection{Endemic equilibrium}

In this section we discuss the existence of endemic equilibria and its stability conditions. Let $P^{*}=$ $\left(S_{h}^{*}, E_{h}^{*}, I_{h}^{*}, H_{h}^{*}, R_{h}^{*}, S_{c}^{*}, I_{c}^{*}\right)$ be an arbitrary endemic equilibrium of the system (1). Therefore, equating the right hand sides of the equations of system (1) to zero, we have

$$
\begin{aligned}
S_{h}^{*}= & \frac{1}{\mu_{h}}\left[\Pi_{h}-\left(\gamma+\mu_{h}\right) E_{h}^{*}\right] \\
I_{h}^{*}= & \frac{r \gamma_{h}}{\mu_{h}+k_{1}+\eta_{1}} E_{h}^{*} \\
H_{h}^{*}= & \frac{1}{b}\left\{(1-r) \gamma_{h}+\frac{\eta_{1} r \gamma_{h}}{\mu_{h}+k_{1}+\eta_{1}}\right\} E_{h}^{*} \\
R_{h}^{*}= & \frac{1}{\mu_{h}}\left[\frac{k_{1} r \gamma_{h}}{\mu_{h}+k_{1}+\eta_{1}}\right. \\
& \left.+\frac{k_{2}}{b}\left\{(1-r) \gamma_{h}+\frac{\eta_{1} r \gamma_{h}}{\mu_{h}+k_{1}+\eta_{1}}\right\}\right] E_{h}^{*} \\
S_{c}^{*}= & \frac{\frac{\Pi_{c}}{\mu_{h}}\left[\Pi_{h}-\frac{\delta}{b}\left\{(1-r) \gamma_{h}+\frac{\eta_{1} r \gamma_{h}}{\mu_{h}+k_{1}+\eta_{1}}\right\} E_{h}^{*}\right]}{\frac{\mu_{h}+k_{1}+\eta_{1}}{\mu_{h}}+\frac{\mu_{c}}{\mu_{h}}\left[\Pi_{h}-\frac{\delta}{b}\left\{(1-r) \gamma_{h}+\frac{\eta_{1} r \gamma_{h}}{\mu_{h}+k_{1}+\eta_{1}}\right\} E_{h}^{*}\right]} \\
I_{c}^{*}= & \frac{\beta_{c} r \gamma_{h}}{\mu_{h}+k_{1}+\eta_{1}} E_{h}^{*}+\frac{\mu_{c}}{\mu_{h}}\left[\Pi_{h}-\frac{\delta}{b}\left\{(1-r) \gamma_{h}+\frac{\eta_{1} r \gamma_{h}}{\mu_{h}+k_{1}+\eta_{1}}\right\} E_{h}^{*}\right] \\
N_{h}^{*}= & \frac{1}{\mu_{h}}\left[\Pi_{h}-\frac{\delta}{b}\left\{(1-r) \gamma_{h}+\frac{\eta_{1} r \gamma_{h}}{\mu_{h}+k_{1}+\eta_{1}}\right\} E_{h}^{*}\right]
\end{aligned}
$$

and $E_{h}^{*}$ comes from the equation

$$
f\left(E_{h}^{*}\right)=Q_{1} E_{h}^{* 2}+Q_{2} E_{h}^{*}+Q_{3}=0
$$

where,

$$
\begin{aligned}
Q_{1}= & \frac{c \beta_{h}}{\mu_{h}}\left(\frac{r \gamma_{h}}{a}+d \alpha\right)\left(\frac{\mu_{c} \delta d}{\mu_{h}}-\frac{\beta_{c} r \gamma_{h}}{a}\right) \\
& -\frac{c}{\mu_{h}}\left(\frac{\mu_{c} \delta d}{\mu_{h}}-\frac{\beta_{c} r \gamma_{h}}{a}\right) \delta d \\
Q_{2}= & \frac{\beta_{h} \Pi_{h}}{\mu_{h}}\left(\frac{r \gamma_{h}}{a}+d \alpha\right)\left[\left(\frac{\beta_{c} r \gamma_{h}}{a}-\frac{\mu_{c} \delta d}{\mu_{h}}\right)-\frac{c \mu_{c}}{\mu_{h}}\right] \\
& -\frac{\beta_{d} \beta_{c} \Pi_{c} r \gamma_{h} c}{\mu_{h} \mu_{c} a}-\frac{\Pi_{h} c}{\mu_{h}}\left(\frac{\beta_{c} r \gamma_{h}}{a}-\frac{\mu_{c} \delta d}{\mu_{h}}\right) \\
& +\frac{\Pi_{h} c \mu_{c} \delta d}{\mu_{h}^{2}} \\
Q_{3}= & \frac{\Pi_{h}^{2} c \mu_{c}}{\mu_{h}^{2}}\left(R_{01}-1\right)
\end{aligned}
$$

Here

$$
\begin{aligned}
& a=\mu_{h}+k_{1}+\eta_{1}, \\
& b=\mu_{h}+k_{2}+\delta, \\
& c=\mu_{h}+\gamma_{h}, \\
& d=\frac{1}{b}\left\{(1-r) \gamma_{h}+\frac{\eta_{1} r \gamma_{h}}{\mu_{h}+k_{1}+\eta_{1}}\right\} .
\end{aligned}
$$

Therefore, if $R_{01}>1$, then endemic equilibrium exists.

Now $E_{h}^{*}=\frac{-Q_{2} \pm \sqrt{Q_{2}^{2}-4 Q_{1} Q_{3}}}{2 Q_{1}}$

Case I If $Q_{2}^{2}-4 Q_{1} Q_{3}>0$, there may exist two endemic equilibrium

Case II If $Q_{2}^{2}-4 Q_{1} Q_{3}=0$, there may exists unique endemic equilibrium.

Case III If $Q_{2}^{2}-4 Q_{1} Q_{3}<0$, there exist no endemic equilibrium..

Now from the Tables 3 and 4, it is clear from Case (II) of both table that, the model (2) has a unique endemic equilibrium whenever $R_{01}>1$ and $R_{01}<1$. In case (I) in Table 4, we observed that, if $Q_{1}<0, Q_{2}>0$ and $Q_{3}<0$ then the model (2) may have the possibility of backward bifurcation where stable disease-free equilibrium coexists with a stable endemic equilibrium whenever the basic reproduction number $R_{01}$ is less than unity. We explore the details analysis of backward bifurcation in the next section. This phenomenon is illustrated numerically by simulation of the model (2)(See Fig. 2).

\subsubsection{Backward bifurcation analysis}

In this section, we explore the phenomenon of backward bifurcation in system (1). First, we carry out bifurcation analysis by applying the Center Manifold Theorem [4]. Let $x=\left(x_{1}, x_{2}, x_{3}, x_{4}, x_{5}, x_{7}\right)^{T}=$ $\left(S_{h}, E_{1}, I_{1}, H_{1}, R_{h}, S_{c}, I_{c}\right)^{T}$. Thus, the model (2) can be re-written in the form $\frac{d x}{\mathrm{~d} t}=f(x)$, with $f(x)=$ $\left(f_{1}(x), \ldots, f_{7}(x)\right)$, as follows:

$$
\begin{aligned}
\frac{d x_{1}}{\mathrm{~d} t}= & f_{1}=\Pi_{h}-\frac{\beta_{1} x_{1}\left(x_{3}+\alpha_{1} x_{4}\right)}{x_{1}+x_{2}+x_{3}+x_{4}+x_{5}} \\
& -\frac{\beta_{d} x_{1} x_{7}}{x_{1}+x_{2}+x_{3}+x_{4}+x_{5}}-\mu_{h} x_{1} \\
\frac{d x_{2}}{\mathrm{~d} t}= & f_{2}=\frac{\beta_{1} x_{1}\left(x_{3}+\alpha_{1} x_{4}\right)}{x_{1}+x_{2}+x_{3}+x_{4}+x_{5}} \\
& -\frac{\beta_{d} x_{1} x_{7}}{x_{1}+x_{2}+x_{3}+x_{4}+x_{5}}-\left(\mu_{h}+\gamma_{1}\right) x_{2}, \\
\frac{d x_{3}}{\mathrm{~d} t}= & f_{3}=r_{1} \gamma_{1} x_{2}-\left(\mu_{h}+k_{11}+\eta_{1}\right) x_{3}, \\
\frac{d x_{4}}{\mathrm{~d} t}= & f_{4}=\left(1-r_{1}\right) \gamma_{1} x_{2}+\eta_{1} x_{3}-\left(\mu_{h}+k_{21}+\delta_{1}\right) x_{4},
\end{aligned}
$$


Table 3 When $R_{01}>1$

\begin{tabular}{lllll}
\hline Case & $Q_{1}$ & $Q_{2}$ & $Q_{3}$ & Number of endemic equilibrium \\
\hline 1 & + & + & + & No endemic equilibrium \\
& + & - & + & Two endemic equilibrium \\
& - & + & + & Unique endemic equilibrium \\
& - & - & + & Unique endemic equilibrium \\
& + & + & + & No endemic equilibrium \\
& + & - & + & Unique endemic equilibrium \\
& - & + & + & Unique endemic equilibrium \\
& - & - & & No endemic equilibrium \\
\hline
\end{tabular}

Table 4 When $R_{01}<1$

\begin{tabular}{ccccc}
\hline Case & $Q_{1}$ & $Q_{2}$ & $Q_{3}$ & Number of endemic equilibrium \\
\hline 1 & + & + & - & Unique endemic equilibrium \\
& + & - & - & Unique endemic equilibrium \\
& - & + & - & Two endemic equilibrium \\
& - & - & - & No endemic equilibrium \\
& + & - & - & No endemic equilibrium \\
& + & + & - & Unique endemic equilibrium \\
& - & - & & Unique endemic equilibrium \\
& - & &
\end{tabular}

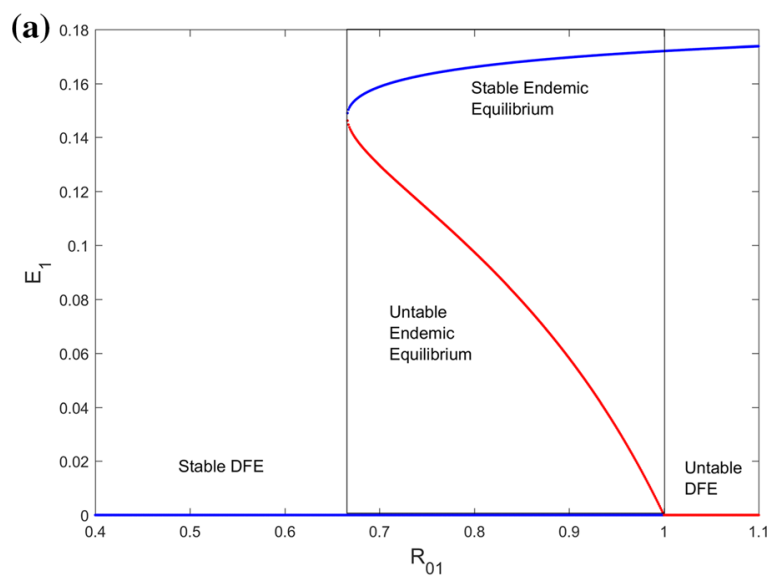

Fig. 2 Backward bifurcation diagram and related time series for the single strain model 2 . The region in the box is the bistable zone. The following set of hypothetical parameter values are

$$
\begin{aligned}
& \frac{d x_{5}}{\mathrm{~d} t}=f_{5}=k_{11} x_{3}+k_{21} x_{4}-\mu_{h} x_{5}, \\
& \frac{d x_{6}}{\mathrm{~d} t}=f_{6}=\Pi_{c}-\frac{\beta_{3} x_{6} x_{3}}{x_{1}+x_{2}+x_{3}+x_{4}+x_{5}}-\mu_{c} x_{6},
\end{aligned}
$$

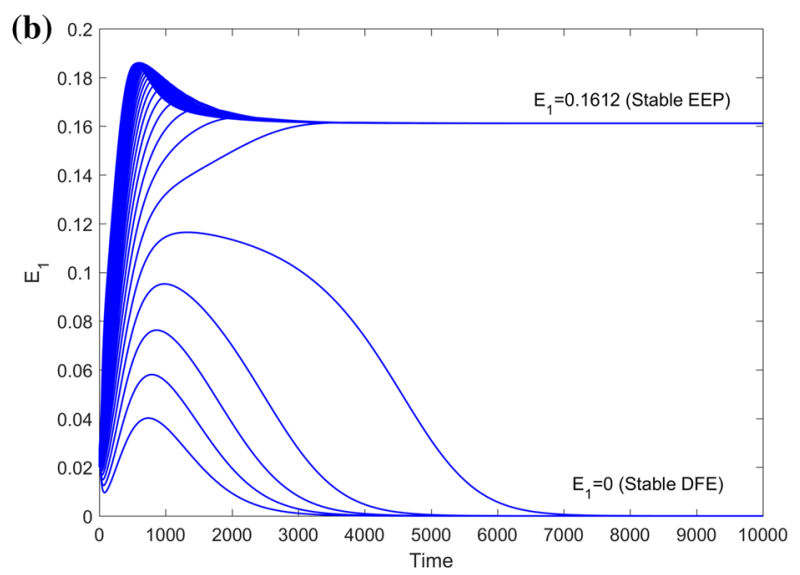

used: $\Pi_{h}=0.007, \Pi_{c}=0.4817, \mu_{h}=0.00154, \mu_{c}=0.009, \beta_{h}=$ $0.200, \beta_{d}=0.0025, \beta_{c}=0.17, \alpha=0.005, \gamma_{h}=0.035, r=0.98$, $k_{1}=0.001, k_{2}=0.0039, \eta_{1}=1.01$ and $\delta=0.04$

$\frac{d x_{7}}{\mathrm{~d} t}=f_{7}=\frac{\beta_{3} x_{6} x_{3}}{x_{1}+x_{2}+x_{3}+x_{4}+x_{5}}-\mu_{c} x_{7}$.

The Jacobian of the system (6) at the DFE $P_{0}$ is given as, 
$J_{P_{0}}=\left(\begin{array}{ccccccc}-\mu_{h} & 0 & -\beta_{1} & -\alpha_{1} \beta_{1} & 0 & 0 & -\beta_{d} \\ 0 & -\left(\mu_{h}+\gamma_{1}\right) & \beta_{1} & \alpha_{1} \beta_{1} & 0 & 0 & \beta_{d} \\ 0 & r_{1} \gamma_{1} & -\left(\mu_{h}+k_{11}+\eta_{1}\right) & 0 & 0 & 0 & 0 \\ 0 & \left(1-r_{1}\right) \gamma_{1} & \eta_{1} & -\left(\mu_{h}+k_{21}+\delta_{1}\right) & 0 & 0 & 0 \\ 0 & 0 & k_{11} & k_{21} & -\mu_{h} & 0 & 0 \\ 0 & 0 & -\frac{\beta_{3} \Pi_{c} \mu_{h}}{\Pi_{h} \mu_{c}} & 0 & 0 & -\mu_{c} & 0 \\ 0 & 0 & \frac{\beta_{3} \Pi_{c} \mu_{h}}{\Pi_{h} \mu_{c}} & 0 & 0 & 0 & -\mu_{c}\end{array}\right)$,

Choose $\beta_{1}$ as the bifurcation parameter, then setting $R_{01}=1$ gives

$$
\begin{aligned}
\beta_{1} & =\beta_{1}^{*} \\
& =\frac{a b c\left(\mu_{c}^{2} \Pi_{h} a c-\beta_{3} \beta_{d} r_{1} \gamma_{1} \Pi_{c} \mu_{h}\right)}{\mu_{c}^{2} \Pi_{h} a c\left(r_{1} \gamma_{1} b+\alpha_{1} r_{1} \gamma_{1} \eta_{1}+\alpha_{1}\left(1-r_{1}\right) \gamma_{1} a\right)}
\end{aligned}
$$

The system (6) at the DFE $P_{0}$ evaluated for $\beta_{1}=\beta_{1}^{*}$ has a simple zero eigenvalue and all other eigenvalues having negative real parts. Hence, we apply the Center Manifold Theorem to analyze the dynamics of (6) near $\beta_{1}=\beta_{1}^{*}$.

The Jacobian of (6) at $\beta_{1}=\beta_{1}^{*}$ denoted by $J_{P_{0}} \mid \beta_{1}=\beta_{1}^{*}$ has a right eigenvector (associated with the zero eigenvalue) given by $w=\left(w_{1}, w_{2}, w_{3}, w_{4}, w_{5}\right.$, $\left.w_{6}, w_{7}\right)^{T}$, where

$$
\begin{aligned}
w_{1} & =-\frac{\left(\mu_{h}+\gamma_{1}\right)}{\mu_{h}} w_{2}, w_{2}=w_{2}>0, \\
w_{3} & =\frac{r_{1} \gamma_{1}}{\left(\mu_{h}+k_{11}+\eta_{1}\right)} w_{2}, \\
w_{4} & =\frac{\left(1-r_{1}\right) \gamma_{1}\left(\mu_{h}+k_{11}+\eta_{1}\right)+\eta_{1} r_{1} \gamma_{1}}{\left(\mu_{h}+k_{11}+\eta_{1}\right)\left(\mu_{h}+k_{21}+\delta_{1}\right)} w_{2}, \\
w_{5} & =\frac{k_{11} w_{3}+k_{21} w_{4}}{\mu_{h}}, \\
w_{6} & =-\frac{\beta_{3} \Pi_{c} \mu_{h} r_{1} \gamma_{1}}{\Pi_{h} \mu_{c}^{2}\left(\mu_{h}+k_{11}+\eta_{1}\right)} w_{2}, \\
w_{7} & =\frac{\beta_{3} \Pi_{c} \mu_{h} r_{1} \gamma_{1}}{\Pi_{h} \mu_{c}^{2}\left(\mu_{h}+k_{11}+\eta_{1}\right)} w_{2} .
\end{aligned}
$$

Similarly, from $J_{P_{0}} \mid \beta_{1}=\beta_{1}^{*}$, we obtain a left eigenvector $v=\left(v_{1}, v_{2}, v_{3}, v_{4}, v_{5}, v_{6}, v_{7}\right)^{T}$ (associated with the zero eigenvalue), where

$$
\begin{aligned}
v_{1} & =0, v_{2}=v_{2}>0, v_{3} \\
& =\frac{1}{\left(\mu_{h}+k_{11}+\eta_{1}\right)}\left[\beta_{1}+\frac{\alpha_{1} \beta_{1} \eta_{1}}{\left(\mu_{h}+k_{21}+\delta_{1}\right)}\right.
\end{aligned}
$$

$$
\begin{gathered}
\left.+\frac{\beta_{d} \Pi_{c} \mu_{h}}{\Pi_{h} \mu_{c}^{2}}\right] v_{2}, \\
v_{4}=\frac{\alpha_{1} \beta_{1}}{\left(\mu_{h}+k_{21}+\delta_{1}\right)} v_{2}, v_{5}=0, v_{6}=0, v_{7}=\frac{\beta_{d}}{\mu_{c}} v_{2} .
\end{gathered}
$$

To show the existence of backward bifurcation, we calculate the following second-order partial derivatives of $f_{i}$ at the disease-free equilibrium $\varepsilon_{0}$ and obtain

$$
\begin{aligned}
\frac{\partial f_{2}}{\partial x_{3} \partial x_{2}}= & \frac{\partial f_{2}}{\partial x_{2} \partial x_{3}}=\frac{\partial f_{2}}{\partial x_{5} \partial x_{3}} \\
& =\frac{\partial f_{2}}{\partial x_{3} \partial x_{5}} \\
& =-\frac{\beta_{1} \mu_{h}}{\Pi_{h}}, \frac{\partial f_{2}}{\partial x_{7} \partial x_{2}}=\frac{\partial f_{2}}{\partial x_{7} \partial x_{3}}=\frac{\partial f_{2}}{\partial x_{7} \partial x_{4}} \\
& =\frac{\partial f_{2}}{\partial x_{7} \partial x_{5}}=\frac{\partial^{2} f_{2}}{\partial^{2} x_{2} \partial x_{6}}=\frac{\partial^{2} f_{2}}{\partial x_{3} \partial x_{6}} \\
& =\frac{\partial^{2} f_{2}}{\partial x_{4} \partial x_{6}}=\frac{\partial^{2} f_{2}}{\partial x_{5} \partial x_{6}}=-\frac{\beta_{d} \mu_{h}}{\Pi_{h}}, \frac{\partial^{2} f_{2}}{\partial x_{3} \partial x_{3}} \\
& =-\frac{2 \beta_{1} \mu_{h}}{\Pi_{h}}, \frac{\partial^{2} f_{2}}{\partial x_{4} \partial x_{2}}=\frac{\partial^{2} f_{2}}{\partial x_{2} \partial x_{4}} \\
& =\frac{\partial^{2} f_{2}}{\partial x_{5} \partial x_{4}}=\frac{\partial^{2} f_{2}}{\partial x_{4} \partial x_{5}}=-\frac{\alpha_{1} \beta_{1} \mu_{h}}{\Pi_{h}}, \frac{\partial^{2} f_{2}}{\partial x_{4} \partial x_{4}} \\
& =-\frac{2 \alpha_{1} \beta_{1} \mu_{h}}{\Pi_{h}}, \frac{\partial^{2} f_{2}}{\partial x_{4} \partial x_{3}}=-\left(\frac{\beta_{1} \mu_{h}}{\Pi_{h}}+\frac{\alpha_{1} \beta_{1} \mu_{h}}{\Pi_{h}}\right) \frac{\partial^{2} f_{7}}{\partial x_{3} \partial x_{1}} \\
& =\frac{\partial^{2} f_{7}}{\partial x_{3} \partial x_{2}}=\frac{\partial^{2} f_{7}}{\partial x_{1} \partial x_{3}}=\frac{\partial^{2} f_{7}}{\partial x_{2} \partial x_{3}} \\
& =\frac{\partial^{2} f_{7}}{\partial x_{4} \partial x_{3}}=\frac{\partial^{2} f_{7}}{\partial x_{5} \partial x_{3}}=-\frac{\beta_{3} \Pi_{c} \mu_{h}}{\Pi_{h} \mu_{c}}, \\
& \frac{\partial^{2} f_{7}}{\partial x_{3} \partial x_{3}}=-\frac{2 \beta_{3} \Pi_{c} \mu_{h}}{\Pi_{h} \mu_{c}}, \frac{\partial^{2} f_{7}}{\partial x_{6} \partial x_{3}} \\
= & \frac{\partial^{2} f_{7}}{\partial x_{3} \partial x_{6}}=\frac{\beta_{3} \mu_{h}}{\Pi_{h}}, \frac{\partial^{2} f_{7}}{\partial x_{3} \partial x_{4}}=\frac{\partial^{2} f_{7}}{\partial x_{3} \partial x_{5}} \\
= & -\frac{\beta_{3} \Pi_{c} \mu_{h}}{\Pi_{h} \mu_{c}}
\end{aligned}
$$

Now we calculate the coefficients $a$ and $b$ defined in Theorem 4.1 [4] of Castillo-Chavez and Song as follow 


$$
\begin{aligned}
a= & \sum_{k, i, j=1}^{6} v_{k} w_{i} w_{j} \frac{\partial^{2} f_{k}\left(0, \beta^{*}\right)}{\partial x_{i} \partial x_{j}} \\
= & \frac{2 \Pi_{c} \mu_{h} v_{7} \beta_{3}}{\Pi_{h} \mu_{c}} \\
& \times\left(w_{3} w_{6}-w_{3}\left(w_{1}+w_{2}+w_{3}+w_{4}+w_{5}\right)\right) \\
& -\frac{2 \beta_{1} v_{2} \mu_{h}}{\Pi_{h}}\left(w_{2} w_{3}+w_{3} w_{3}\right. \\
& +w_{2} w_{4}+\left(1+\alpha_{1}\right) w_{3} w_{4}+w_{3} w_{5} \\
& \left.+\alpha_{1} w_{4} w_{4}++\alpha_{1} w_{4} w_{5}\right) \\
& -\frac{2 v_{2} \mu_{h}}{\Pi_{h}} \beta_{d} w_{7}\left(w_{2}+w_{3}+w_{4}+w_{5}\right)
\end{aligned}
$$

and

$b=\sum_{k, i=1}^{6} v_{k} w_{i} \frac{\partial^{2} f_{k}(0,0)}{\partial x_{i} \partial \beta}=v_{2}\left(w_{3}+\alpha_{1} w_{4}\right)>0$.

Since the coefficient $b$ is always positive, system (1) undergoes backward bifurcation at $R_{01}=1$, if $a>0$, namely if

\section{Two strain model analysis}

4.1 Diseases-free equilibrium and basic reproduction number

The diseases-free equilibrium can be obtained for the system (2) by putting $E_{1}=0, E_{2}=0, I_{1}=0, I_{2}=$ $0, H_{1}=0, H_{2}=0, I_{c}=0$, which is denoted by $P_{01}=$ $\left(S_{h}, 0,0,0,0,0,0, R_{h}, S_{c}, 0\right)$, where

$S_{h}=\frac{\Pi_{h}}{\mu_{h}}, S_{c}=\frac{\Pi_{c}}{\mu_{c}}, R_{h}=0$.

\subsubsection{Basic reproduction number}

The basic reproduction number has been computed by next generation approach [16]. It is defined as the average number of secondary infections produced by a single infected individual in a susceptible population and it is given as

$R_{0}=\max \left\{R_{01}, R_{02}\right\}$

where

$\beta_{3}>\frac{\mu_{c} \beta_{1} v_{2}\left(w_{2} w_{3}+w_{3} w_{3}+w_{2} w_{4}+\left(1+\alpha_{1}\right) w_{3} w_{4}+w_{3} w_{5}+\alpha_{1} w_{4} w_{4}+\alpha_{1} w_{4} w_{5}\right)+\mu_{c} v_{2} \beta_{d} w_{7}\left(Z_{w}-w_{1}\right)}{v_{7} \beta_{3} \Pi_{c}\left(w_{3} w_{6}-w_{3} Z_{w}\right)}$,

where, $Z_{w}=w_{1}+w_{2}+w_{3}+w_{4}+w_{5}$.

We have established the following conclusion.

Theorem 3 System (1) undergoes a backward bifurcation at $R_{01}=1$ whenever the inequality 13 holds.

We numerically shown the existence of backward bifurcation for the model (2), which verifies our analytical finding. The bifurcation diagram and related time series is presented in Fig. 2.

This implies that even if the basic reproduction number is less than unity, the disease may not be eradicated. To ensure the global stability of DFE one must reduce $R_{01}$ below a certain threshold namely, $R_{01}^{c}$.

$$
\begin{aligned}
R_{0 i}= & \frac{\beta_{i} r_{i} \gamma_{i}}{\left(\mu_{h}+\gamma_{i}\right)\left(\mu_{h}+k_{1 i}+\eta_{1}\right)} \\
& +\frac{\alpha_{i} \beta_{i} r_{i} \gamma_{i} \eta_{i}}{\left(\mu_{h}+\gamma_{i}\right)\left(\mu_{h}+k_{1 i}+\eta_{i}\right)\left(\mu_{h}+k_{2 i}+\delta_{i}\right)} \\
& +\frac{\alpha_{i} \beta_{i}\left(1-r_{i}\right) \gamma_{i}}{\left(\mu_{h}+\gamma_{i}\right)\left(\mu_{h}+k_{2 i}+\delta_{i}\right)} \\
& +\frac{\beta_{i+2} \beta_{d} r_{i} \gamma_{i} \Pi_{c} \mu_{h}}{\mu_{c}^{2} \Pi_{h}\left(\mu_{h}+\gamma_{i}\right)\left(\mu_{h}+k_{1 i}+\eta_{i}\right)}
\end{aligned}
$$


(a)

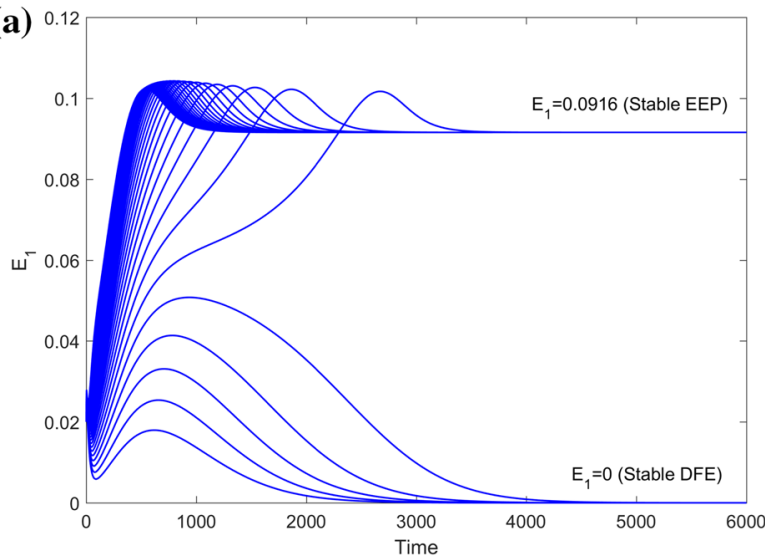

(b)

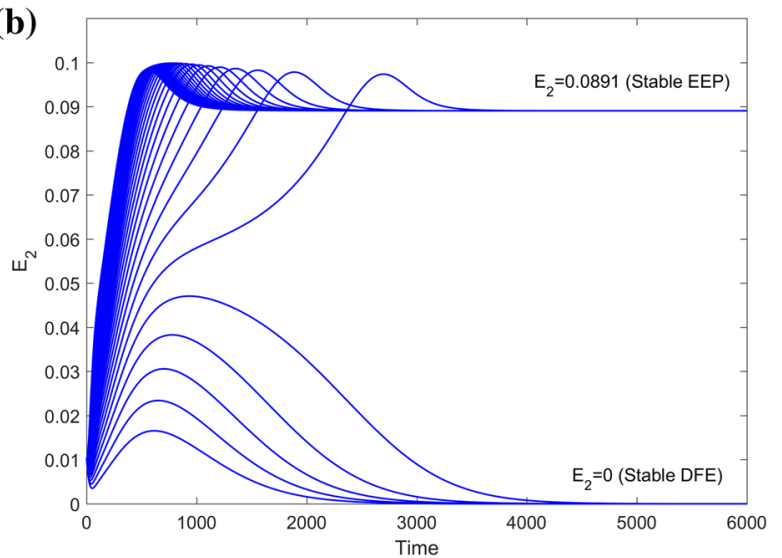

Fig. 3 Time series for the full model 1 showing bistability of the equilibrium values

Table 5 Normalized sensitivity indices

\begin{tabular}{llllll}
\hline$X_{R_{0}}^{\beta_{1}}$ & $X_{R_{0}}^{\beta_{3}}$ & $X_{R_{0}}^{\beta_{d}}$ & $X_{R_{0}}^{r_{1}}$ & $X_{R_{0}}^{k_{1} 1}$ & $X_{R_{0}}^{k_{2} 1}$ \\
\hline 0.0431 & 0.9569 & 0.9568 & 0.9392 & -0.9797 & -0.0183
\end{tabular}

\subsection{Backward bifurcation analysis of the full model}

We could not analytically find the conditions for the existence of backward bifurcation in case of the full model. Thus, we checked numerically whether this phenomenon occurs for the full model or not. Interestingly, we find parameters for which the full model undergoes bistable dynamics even if the basic reproduction number is below unity. The time series of bistability is depicted in Fig. 3 .

The following set of hypothetical parameter values are used: $\Pi_{h}=0.007, \Pi_{c}=0.4817, m=0.5, \alpha_{1}=\alpha_{2}=$ $0.0005 ; \gamma_{1}=\gamma_{2}=0.035 ; \mu_{h}=0.00154, \mu_{c}=0.009$, $\beta_{1}=0.2 ; \beta_{2}=0.17 ; \beta_{3}=0.2 ; \beta_{4}=0.17 ; \beta_{d}=0.0025$; $r_{1}=0.98, r_{2}=0.7 ; k_{11}=k_{21}=0.001, k_{12}=0.0039$, $k_{22}=0.0017 \eta_{1}=\eta_{2}=1.01$ and $\delta_{1}=\delta_{2}=0.04$. For this parameter values, the basic reproduction number is found to be $0.7941<1$. This indicates that backward bifurcation or existence of bistability for $R_{0}<1$ is an inherent property of MERS-CoV transmission dynamics. In turn, this point out that the control efforts will need more careful considerations.

\subsection{Normalized sensitivity analysis}

Due to the complex dynamical behavior of the model, we calculate the normalized sensitivity indices of the model parameters with respect to $R_{0}$ in order to find the most influential parameters. We consider the following parameters for this analysis: $\beta_{1}, \beta_{2}, \beta_{3}, \beta_{4}, \beta_{d}, r_{1}, r_{2}$, $k_{11}, k_{12}, k_{21}$ and $k_{22}$. We use the parameter values from Table 2 for the baseline values. However, the mathematical definition of the normalized forward sensitivity index of a variable $z$ with respect to a parameter $\tau$ (where $z$ depends explicitly on the parameter $\tau$ ) is given as:

$X_{z}^{\tau}=\frac{\partial z}{\partial \tau} \times \frac{\tau}{z}$.

It is important to note that we used the following expression for $R_{0}: R_{0}=\frac{R_{01}+R_{02}+\left|R_{01}-R_{02}\right|}{2}$. The sensitivity indices of $R_{0}$ with respect to the most important parameters are given in Table 5.

All other parameters have sensitivity indices less than 0.001 . However, the fact that $X_{R_{0}}^{\beta_{1}}=0.0431$ means that if we increase $1 \%$ in $\beta_{1}$, keeping other parameters fixed, will produce $0.0431 \%$ increase in $R_{c}$. Similarly, $X_{R_{0}}^{k_{2} 1}=-0.0183$ means increasing the parameter $k_{2} 1$ by $1 \%$, the value of $R_{0}$ will be decreased by $0.0183 \%$ keeping the value of other parameters be fixed. Biologically, this indicates that the transmission rate from 
strain-1 infected humans to susceptible camels and transmission rate from camels to susceptible humans are very crucial. These two quantities can be decreased by using personal protection measures. On the other hand, the recovery rate of un-notified infected with strain-1 should be increased to reduce the $R_{0}$ below unity. This can be achieved by tracing and treating unnotified infected humans with strain-1.

\subsection{Optimal control analysis}

In countries such as Saudi Arabia, Republic of Korea, United Arab Emirates etc, MERS-CoV is of great concern. For MERS-CoV transmission dynamics we formulate an optimal control problem [25]. The goal here is to demonstrate that time-dependent anti-MERS control strategies that can be implemented while minimizing the cost of implementing these measures. There are several control mechanisms that are put in place to minimize disease transmission. We consider four types of individual control mechanisms to mitigate MERS-CoV in the community. These single strategies are explained below:

1. Personal protection $\left(u_{1}(t)\right)$ : Preventing transmission of MERS-CoV in society requires the application of personal protective measures such as the use of face masks [7]. In this strategy, the susceptible humans are expected to reduce contacts with infected humans and camels.

2. Isolation of strain-1 infected notified people $\left(u_{2}(t)\right)$ : In this control mechanism, the strain-1 infected people who are notified are isolated from the other patients. These patients are given special attention so as to reduce contact with other people.

3. Isolation of strain-2 infected notified people $\left(u_{3}(t)\right)$ : Similarly, strain-2 infected people who are notified are specially isolated.

4. Isolation of infected camels $\left(u_{4}(t)\right)$ : Infected camels are to be found and are to be isolated in a special healthcare setting.

Note that for bounded Lebesgue measurable controls and non-negative initial conditions, non-negative bounded solutions to the state system exist [22]. The control functions $u_{1}(t), u_{2}(t), u_{3}(t)$ and $u_{4}(t)$ are bounded and Lebesgue integrable functions, where the associated force of infection in the human population is reduced by a factor of $\left(1-u_{1}(\mathrm{t})\right)$, where $u_{1}(\mathrm{t})$ measures the level of successful prevention efforts and the control functions $u_{2}(\mathrm{t}), u_{3}(\mathrm{t})$ and $u_{4}(\mathrm{t})$ represent the isolation of notified humans infected with strain-1 or strain-2 and isolation of infected camels, respectively.

Taking the above-mentioned interventions into account, the model (1) takes the following form

$$
\begin{aligned}
\frac{\mathrm{d} S_{h}}{\mathrm{~d} t}= & \Pi_{h}-\beta_{1} \frac{\left(1-u_{1}\right) S_{h}}{N_{h}}\left(I_{1}+\alpha_{1} H_{1}\right) \\
& -\beta_{2} \frac{\left(1-u_{1}\right) S_{h}}{N_{h}}\left(I_{2}+\alpha_{2} H_{2}\right) \\
& -\beta_{d} \frac{\left(1-u_{1}\right) S_{h}}{N_{h}} I_{c}-\mu_{h} S_{h},
\end{aligned}
$$

$$
\begin{aligned}
\frac{\mathrm{d} E_{1}}{\mathrm{~d} t}= & \beta_{1} \frac{\left(1-u_{1}\right) S_{h}}{N_{h}}\left(I_{1}+\alpha_{1} H_{1}\right) \\
& +m \beta_{d} \frac{\left(1-u_{1}\right) S_{h}}{N_{h}} I_{c}-\mu_{h} E_{1}-\gamma_{1} E_{1},
\end{aligned}
$$

$\frac{\mathrm{d} E_{2}}{\mathrm{~d} t}=\beta_{2} \frac{\left(1-u_{1}\right) S_{h}}{N_{h}}\left(I_{2}+\alpha_{2} H_{2}\right)$

$$
+(1-m) \beta_{d} \frac{\left(1-u_{1}\right) S_{h}}{N_{h}} I_{c}-\mu_{h} E_{2}-\gamma_{2} E_{2},
$$

$\frac{\mathrm{d} I_{1}}{\mathrm{~d} t}=r_{1} \gamma_{1} E_{1}-\left(\mu_{h}+k_{11}+\eta_{1}\right) I_{1}$,

$\frac{\mathrm{d} I_{2}}{\mathrm{~d} t}=r_{2} \gamma_{2} E_{2}-\left(\mu_{h}+k_{12}+\eta_{2}\right) I_{2}$,

$\frac{\mathrm{d} H_{1}}{\mathrm{~d} t}=\left(1-r_{1}\right) \gamma_{1} E_{1}+\eta_{1} I_{1}$

$-\left(\mu_{h}+k_{21}+\delta_{1}\right) H_{1}-u_{2} H_{1}$,

$\frac{\mathrm{d} H_{2}}{\mathrm{~d} t}=\left(1-r_{2}\right) \gamma_{2} E_{2}+\eta_{2} I_{2}$

$$
-\left(\mu_{h}+k_{22}+\delta_{2}\right) H_{2}-u_{3} H_{2},
$$

$\frac{\mathrm{d} R_{h}}{\mathrm{~d} t}=k_{11} I_{1}+k_{12} I_{2}+k_{21} H_{1}+k_{22} H_{2}-\mu_{h} R_{h}$,

$\frac{\mathrm{d} S_{c}}{\mathrm{~d} t}=\Pi_{c}-\beta_{3} \frac{S_{c} I_{1}}{N_{h}}-\beta_{4} \frac{S_{c} I_{2}}{N_{h}}-\mu_{c} S_{c}$,

$\frac{\mathrm{d} I_{c}}{\mathrm{~d} t}=\beta_{3} \frac{S_{c} I_{1}}{N_{h}}+\beta_{4} \frac{S_{c} I_{2}}{N_{h}}-\mu_{c} I_{c}-u_{4} I_{c}$.

The problem is to minimize the cost function [12, 20,25] given as follows:

$$
\begin{aligned}
J\left(u_{1}, u_{2}, u_{3}, u_{4}\right) \\
=\int_{0}^{T}\left(C_{1} H_{1}+C_{2} H_{2}+C_{3} I_{c}+\frac{1}{2} C_{4} u_{1}^{2}\right. \\
\left.\quad+\frac{1}{2} C_{5} u_{2}^{2}+\frac{1}{2} C_{6} u_{3}^{2}+\frac{1}{2} C_{7} u_{4}^{2}\right) \mathrm{d} t
\end{aligned}
$$

subject to the state system given by (14). The goal is to minimize the hospitalized infected human populations, infected camel population and the cost of implement- 
ing the control. Basically, we assume that the costs are proportional to the square of the corresponding control function. Our goal is to find optimal control functions $\left(u_{1}^{*}, u_{2}^{*}, u_{3}^{*}, u_{4}^{*}\right)$ such that

$$
\begin{aligned}
& J\left(u_{1}^{*}, u_{2}^{*}, u_{3}^{*}, u_{4}^{*}\right) \\
& \quad=\min \left\{J\left(u_{1}, u_{2}, u_{3}, u_{4}\right) \mid\left(u_{1}, u_{2}, u_{3}, u_{4}\right) \in \Gamma\right\}
\end{aligned}
$$

subject to the system of equations given by (14), where $\Gamma=\left\{\left(u_{1}, u_{2}, u_{3}, u_{4}\right) \mid u_{i}(t)\right.$ is Lebesgue measurable on $[0, T], 0$

$$
\left.\leq u_{i}(t) \leq b_{i}, i=1,2,3,4\right\}
$$

is the control set.

Pontryagin's maximum principle [20] can be applied to find the optimal rates of different interventions and layered combinations. This principle converts system (14) into a minimizing problem. The Hamiltonian $H$, with respect to $u_{1}, u_{2}, u_{3}$ and $u_{4}$, can be written as:

$$
\begin{aligned}
& H=\left(C_{1} H_{1}+C_{2} H_{2}+C_{3} I_{c}+\frac{1}{2} C_{4} u_{1}^{2}+\frac{1}{2} C_{5} u_{2}^{2}\right. \\
& \left.+\frac{1}{2} C_{6} u_{3}^{2}+\frac{1}{2} C_{7} u_{4}^{2}\right) \\
& +\lambda_{1}\left[\Pi_{h}-\beta_{1} \frac{\left(1-u_{1}\right) S_{h}}{N_{h}}\left(I_{1}+\alpha_{1} H_{1}\right)\right. \\
& -\beta_{2} \frac{\left(1-u_{1}\right) S_{h}}{N_{h}}\left(I_{2}+\alpha_{2} H_{2}\right) \\
& \left.-\beta_{d} \frac{\left(1-u_{1}\right) S_{h}}{N_{h}} I_{c}-\mu_{h} S_{h}\right] \\
& +\lambda_{2}\left[\beta_{1} \frac{\left(1-u_{1}\right) S_{h}}{N_{h}}\left(I_{1}+\alpha_{1} H_{1}\right)\right. \\
& \left.+m \beta_{d} \frac{\left(1-u_{1}\right) S_{h}}{N_{h}} I_{c}-\mu_{h} E_{1}-\gamma_{1} E_{1}\right] \\
& +\lambda_{3}\left[\beta_{2} \frac{\left(1-u_{1}\right) S_{h}}{N_{h}}\left(I_{2}+\alpha_{2} H_{2}\right)\right. \\
& \left.+(1-m) \beta_{d} \frac{\left(1-u_{1}\right) S_{h}}{N_{h}} I_{c}-\mu_{h} E_{2}-\gamma_{2} E_{2}\right] \\
& +\lambda_{4}\left[r_{1} \gamma_{1} E_{1}-\left(\mu_{h}+k_{11}+\eta_{1}\right) I_{1}\right] \\
& +\lambda_{5}\left[r_{2} \gamma_{2} E_{2}-\left(\mu_{h}+k_{12}+\eta_{2}\right) I_{2}\right] \\
& +\lambda_{6}\left[\left(1-r_{1}\right) \gamma_{1} E_{1}+\eta_{1} I_{1}\right. \\
& \left.-\left(\mu_{h}+k_{21}+\delta_{1}\right) H_{1}-u_{2} H_{1}\right] \\
& +\lambda_{7}\left[\left(1-r_{2}\right) \gamma_{2} E_{2}+\eta_{2} I_{2}\right. \\
& \left.-\left(\mu_{h}+k_{22}+\delta_{2}\right) H_{2}-u_{3} H_{2}\right] \\
& +\lambda_{8}\left[k_{11} I_{1}+k_{12} I_{2}+k_{21} H_{1}+k_{22} H_{2}-\mu_{h} R_{h}\right]
\end{aligned}
$$

$$
\begin{aligned}
& +\lambda_{9}\left[\Pi_{c}-\beta_{3} \frac{S_{c} I_{1}}{N_{h}}-\beta_{4} \frac{S_{c} I_{2}}{N_{h}}-\mu_{c} S_{c}\right] \\
& +\lambda_{10}\left[\beta_{3} \frac{S_{c} I_{1}}{N_{h}}+\beta_{4} \frac{S_{c} I_{2}}{N_{h}}-\mu_{c} I_{c}-u_{4} I_{c}\right]
\end{aligned}
$$

where $\lambda_{i}, i=1, \ldots ., 10$ are the adjoint variables. In the following theorem, we present the adjoint system and control characterization. To simplify notation, we let $\hbar=\frac{1}{N_{h}}$.

Theorem 4 Given an optimal control $\left(u_{1}^{*}, u_{2}^{*}, u_{3}^{*}, u_{4}^{*}\right)$, and corresponding state solutions $S_{h}, E_{1}, E_{2}, I_{1}, I_{2}$, $H_{1}, H_{2}, R_{h}, S_{c}, I_{c}$ of the corresponding state system (14), there exists adjoint variables, $\lambda_{i}$, for $i=1, \ldots, 10$, satisfying

$$
\begin{aligned}
& \frac{\partial \lambda_{1}}{\partial t}=-\left(\lambda_{2}-\lambda_{1}\right)\left[\beta_{1}\left(1-u_{1}(t)\right)\left(I_{1}+\alpha_{1} H_{1}\right)\left(\hbar-S_{h} \hbar^{2}\right)\right] \\
& -\left(\lambda_{3}-\lambda_{1}\right)\left[\beta_{2}\left(1-u_{1}(t)\right)\left(I_{2}+\alpha_{2} H_{2}\right)\left(\hbar-S_{h} \hbar^{2}\right)\right] \\
& -\left(\lambda_{2} m+\lambda_{3}(1-m)-\lambda_{1}\right) \\
& {\left[\beta_{d}\left(1-u_{1}(t)\right) I_{c}\left(\hbar-S_{h} \hbar^{2}\right)\right]+\lambda_{1} \mu_{h},} \\
& \frac{\partial \lambda_{2}}{\partial t}=-\left(\lambda_{1}-\lambda_{2}\right)\left[\beta_{1}\left(1-u_{1}(t)\right) S_{h}\left(I_{1}+\alpha_{1} H_{1}\right) \hbar^{2}\right] \\
& -\left(\lambda_{1}-\lambda_{3}\right)\left[\beta_{2}\left(1-u_{1}(t)\right) S_{h}\left(I_{2}+\alpha_{2} H_{2}\right) \hbar^{2}\right] \\
& -\left(\lambda_{1}-\lambda_{2} m+\lambda_{3}(1-m)\right)\left[\beta_{d}\left(1-u_{1}(t)\right) S_{h} I_{c} \hbar^{2}\right] \\
& +\lambda_{2}\left(\mu_{h}+\gamma_{1}\right)-\left(\lambda_{4}-\lambda_{6}\right) r_{1} \gamma_{1}-\lambda_{6} \gamma_{1}, \\
& \frac{\partial \lambda_{3}}{\partial t}=-\left(\lambda_{1}-\lambda_{2}\right)\left[\beta_{1}\left(1-u_{1}(t)\right) S_{h}\left(I_{1}+\alpha_{1} H_{1}\right) \hbar^{2}\right] \\
& -\left(\lambda_{1}-\lambda_{3}\right)\left[\beta_{2}\left(1-u_{1}(t)\right) S_{h}\left(I_{2}+\alpha_{2} H_{2}\right) \hbar^{2}\right] \\
& -\left(\lambda_{1}-\lambda_{2} m+\lambda_{3}(1-m)\right)\left[\beta_{d}\left(1-u_{1}(t)\right) S_{h} I_{c} \hbar^{2}\right] \\
& +\lambda_{3}\left(\mu_{h}+\gamma_{2}\right)-\left(\lambda_{5}-\lambda_{7}\right) r_{2} \gamma_{2}-\lambda_{7} \gamma_{2}, \\
& \frac{\partial \lambda_{4}}{\partial t}=-\left(\lambda_{2}-\lambda_{1}\right)\left[\beta_{1}\left(1-u_{1}(t)\right) S_{h}\left(\hbar-\left(I_{1}+\alpha_{1} H_{1}\right) \hbar^{2}\right)\right] \\
& -\left(\lambda_{1}-\lambda_{3}\right)\left[\beta_{2}\left(1-u_{1}(t)\right) S_{h}\left(I_{2}+\alpha_{2} H_{2}\right) \hbar^{2}\right] \\
& -\left(\lambda_{1}-\lambda_{2} m-\lambda_{3}(1-m)\right)\left[\beta_{d}\left(1-u_{1}(t)\right) S_{h} I_{c} \hbar^{2}\right] \\
& +\lambda_{4}\left(\mu_{h}+k_{11}+\eta_{1}\right)-\lambda_{6} \eta_{1}-\lambda_{8} k_{11} \\
& -\left(\lambda_{10}-\lambda_{9}\right)\left[\beta_{3} S_{c}\left(\hbar-I_{1} \hbar^{2}\right)-\beta_{4} S_{c} I_{2} \hbar^{2}\right] \\
& \frac{\partial \lambda_{5}}{\partial t}=-\left(\lambda_{1}-\lambda_{2}\right)\left[\beta_{1}\left(1-u_{1}(t)\right) S_{h}\left(I_{1}+\alpha_{1} H_{1}\right) \hbar^{2}\right] \\
& -\left(\lambda_{3}-\lambda_{1}\right)\left[\beta_{2}\left(1-u_{1}(t)\right) S_{h}\left(\hbar-\left(I_{2}+\alpha_{2} H_{2}\right)\right) \hbar^{2}\right] \\
& -\left(\lambda_{1}-\lambda_{2} m+\lambda_{3}(1-m)\right)\left[\beta_{d}\left(1-u_{1}(t)\right) S_{h} I_{c} \hbar^{2}\right] \\
& +\lambda_{5}\left(\mu_{h}+k_{12}+\eta_{2}\right)-\lambda_{7} \eta_{2}-\lambda_{8} k_{12} \\
& -\left(\lambda_{9}-\lambda_{10}\right)\left[\beta_{3} S_{c} I_{1} \hbar^{2}-\beta_{4} S_{c}\left(\hbar-I_{2} \hbar^{2}\right)\right] \\
& \frac{\partial \lambda_{6}}{\partial t}=-C_{1}-\left(\lambda_{2}-\lambda_{1}\right) \\
& {\left[\beta_{1}\left(1-u_{1}(t)\right) S_{h}\left(\alpha_{1} \hbar-\left(I_{1}+\alpha_{1} H_{1}\right) \hbar^{2}\right)\right]-\left(\lambda_{1}-\lambda_{3}\right)} \\
& {\left[\beta_{2}\left(1-u_{1}(t)\right) S_{h}\left(I_{2}+\alpha_{2} H_{2}\right) \hbar^{2}\right]} \\
& -\left(\lambda_{1}-\lambda_{2} m-\lambda_{3}(1-m)\right)\left[\beta_{d}\left(1-u_{1}(t)\right) S_{h} I_{c} \hbar^{2}\right]
\end{aligned}
$$




$$
\begin{aligned}
& \quad+\lambda_{6}\left[\left(\mu_{h}+k_{21}+\delta_{1}\right)+u_{2}(t)\right]-\lambda_{8} k_{21} \\
& \quad-\left(\lambda_{9}-\lambda_{10}\right)\left[\beta_{3} S_{c} I_{1} \hbar^{2}+\beta_{4} S_{c} I_{2} \hbar^{2}\right] \\
& \frac{\partial \lambda_{7}}{\partial t}=-C_{2}-\left(\lambda_{1}-\lambda_{2}\right)\left[\beta_{1}\left(1-u_{1}(t)\right) S_{h}\left(I_{1}+\alpha_{1} H_{1}\right) \hbar^{2}\right] \\
& \quad-\left(\lambda_{3}-\lambda_{1}\right)\left[\beta_{2}\left(1-u_{1}(t)\right) S_{h}\left(\alpha_{2} \hbar-\left(I_{2}+\alpha_{2} H_{2}\right)\right) \hbar^{2}\right] \\
& -\left(\lambda_{1}-\lambda_{2} m-\lambda_{3}(1-m)\right)\left[\beta_{d}\left(1-u_{1}(t)\right) S_{h} I_{c} \hbar^{2}\right] \\
& \quad+\lambda_{7}\left[\left(\mu_{h}+k_{22}+\delta_{2}\right)+u_{3}(t)\right]-\lambda_{8} k_{22} \\
& -\left(\lambda_{9}-\lambda_{10}\right)\left[\beta_{3} S_{c} I_{1} \hbar^{2}+\beta_{4} S_{c} I_{2} \hbar^{2}\right] \\
& \frac{\partial \lambda_{8}}{\partial t}=-\left(\lambda_{1}-\lambda_{2}\right)\left[\beta_{1}\left(1-u_{1}(t)\right) S_{h}\left(I_{1}+\alpha_{1} H_{1}\right) \hbar^{2}\right] \\
& -\left(\lambda_{1}-\lambda_{3}\right)\left[\beta_{2}\left(1-u_{1}(t)\right) S_{h}\left(I_{2}+\alpha_{2} H_{2}\right) \hbar^{2}\right] \\
& -\left(\lambda_{1}-\lambda_{2} m-\lambda_{3}(1-m)\right)\left[\beta_{d}\left(1-u_{1}(t)\right) S_{h} I_{c} \hbar^{2}\right] \\
& \quad+\lambda_{8} \mu_{h}-\left(\lambda_{9}-\lambda_{10}\right)\left[\beta_{3} S_{c} I_{1} \hbar^{2}+\beta_{4} S_{c} I_{2} \hbar^{2}\right] \\
& \frac{\partial \lambda_{9}}{\partial t}=-\left(\lambda_{10}-\lambda_{9}\right) \\
& \quad\left[\beta_{3} I_{1}\left(\hbar-S_{c} \hbar^{2}\right)+\beta_{4} I_{2}\left(\hbar-S_{c} \hbar\right)\right] \\
& \frac{\partial \lambda_{10}}{\partial t}=-C_{3}-\left(\lambda_{3}(1-m)+\lambda_{2} m-\lambda_{1}\right) \\
& \quad\left[\beta_{d}\left(1-u_{1}(t)\right) S_{h} \hbar\right]+\lambda_{10}\left(\mu_{c}+u_{4}(t)\right) .
\end{aligned}
$$

\section{The terminal conditions are}

$\lambda_{i}(T)=0$ for $i=1, \ldots \ldots, 10$.

Furthermore, the optimal controls $u_{1}^{*}, u_{2}^{*}, u_{3}^{*}, u_{4}^{*}$ and are represented by

$$
\begin{aligned}
u_{1}^{*}= & \max \left\{0, \min \left\{1, \frac{1}{C_{4}}\left[\beta_{1} S_{h} \hbar\left(I_{1}+\alpha_{1} H_{1}\right)\left(\lambda_{2}-\lambda_{1}\right)\right.\right.\right. \\
& +\beta_{2} S_{h} \hbar\left(I_{2}+\alpha_{2} H_{2}\right)\left(\lambda_{3}-\lambda_{1}\right) \\
& \left.\left.\left.+\beta_{d} S_{h} I_{c} \hbar\left(\lambda_{3}(1-m)+\lambda_{2} m-\lambda_{1}\right)\right]\right\}\right\} \\
u_{2}^{*} & =\max \left\{0, \min \left\{1, \frac{1}{C_{5}} \lambda_{6} H_{1}\right\}\right\} \\
u_{3}^{*}= & \max \left\{0, \min \left\{1, \frac{1}{C_{6}} \lambda_{7} H_{2}\right\}\right\} \\
u_{4}^{*} & =\max \left\{0, \min \left\{1, \frac{1}{C_{7}} \lambda_{10} I_{c}\right\}\right\}
\end{aligned}
$$

Proof The adjoint system results from Pontryagin's maximum Principle [20]

$$
\frac{\partial \lambda_{1}}{\partial t}=-\frac{\partial H}{\partial S_{h}}, \frac{\partial \lambda_{2}}{\partial t}=-\frac{\partial H}{\partial E_{1}}, \ldots . ., \frac{\partial \lambda_{10}}{\partial t}=-\frac{\partial H}{\partial I_{c}}
$$

with zero final time conditions (transversality).

To get the characterization of the optimal control given by (20), we solve the equations on the interior of
Table 6 Cost effectiveness of control strategies

\begin{tabular}{lrl}
\hline Strategies & ACER & IAR \\
\hline$u_{1}+u_{4}$ & 0.2793 & 0.0268 \\
$u_{1}+u_{2}+u_{3}$ & 57.6686 & 0.0126 \\
$u_{1}+u_{2}+u_{4}$ & 0.2798 & 0.0264 \\
$u_{1}+u_{3}+u_{4}$ & 0.2815 & 0.0261 \\
$u_{2}+u_{3}+u_{4}$ & 36.4612 & 0.0152 \\
$u_{1}+u_{2}+u_{3}+u_{4}$ & 35.9899 & 0.0153 \\
\hline
\end{tabular}

the control set,

$\frac{\partial H}{\partial u_{1}}=0, \frac{\partial H}{\partial u_{2}}=0, \frac{\partial H}{\partial u_{3}}=0, \frac{\partial H}{\partial u_{4}}=0$

Using the bounds on the controls, we obtain the desired characterization.

The optimality system consists of the state system (14) with the initial conditions, the adjoint system with the terminal conditions and the control characterization.

We now numerically study the optimal strategies for the effect of combined control interventions by using a forward backward sweep fourth order Runge-Kutta method. The objective is to optimize the controls, $u_{1}$, $u_{2}, u_{3}$ and $u_{4}$. The values for the parameters used are given in Table 2 and the cost coefficients are taken as $C 1=300, C_{2}=300, C_{3}=100, C_{4}=50, C_{5}=400$, $C_{6}=400$ and $C_{7}=200$. We consider six scenarios combining the four individual controls. We calculate two quantities to distinguish between interventions strategies, Infection Averted Ratio (IAR) and Average Cost-Effectiveness Ratio (ACER) [25]. IAR and ACER are defined as follows:

$$
\begin{aligned}
& \text { IAR }=\frac{\text { Number of infection averted }}{\text { Number of successfully recovered }}, \\
& \text { ACER }=\frac{\text { Total cost of intervention }}{\text { Number of infection averted }} .
\end{aligned}
$$

These two measures are reported in the Table 6. These values indicate that combination of $u_{1}$ and $u_{4}$ is the best strategy to apply. We further give the graphical representation of the cases and control profiles in Figs. 4 and 5 respectively.

These results indicate that reducing the contacts with infected people as well as infected camels along with isolation of infected camels is a very useful control 
(a)
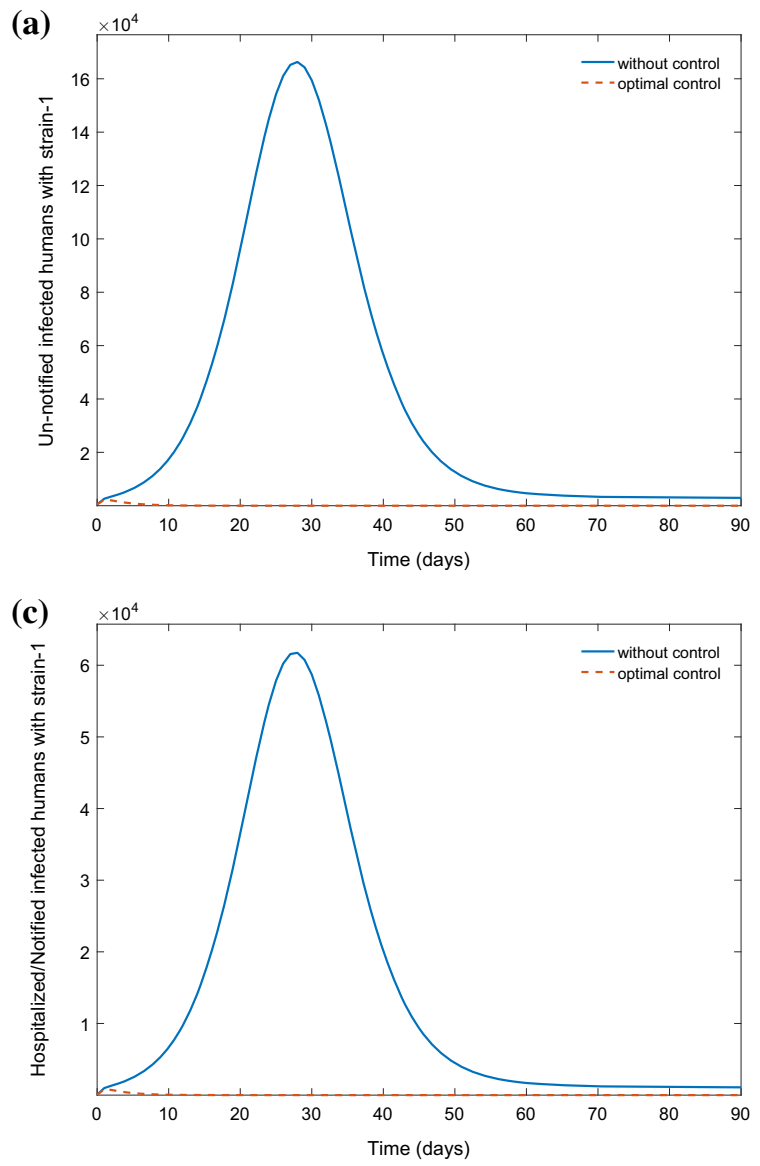

(b)

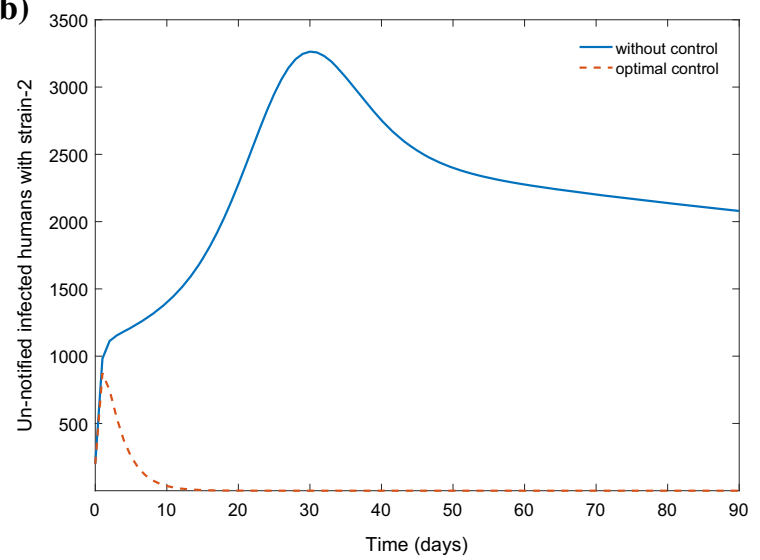

(d)

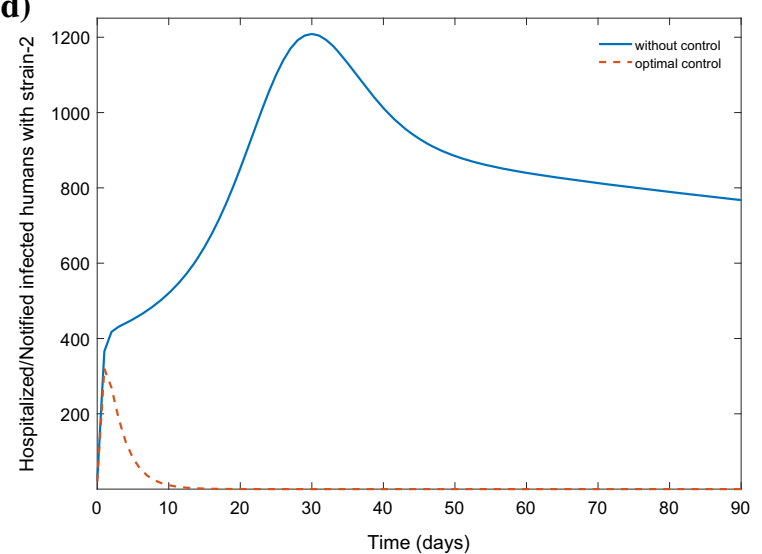

(e)

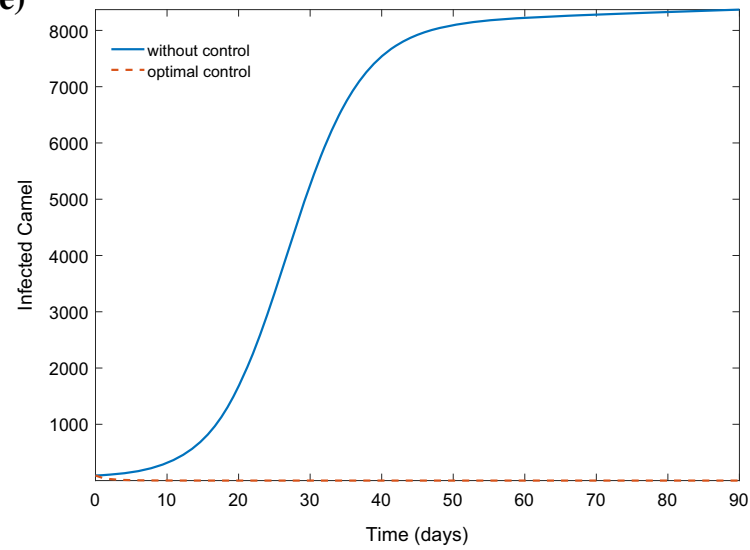

Fig. 4 Time series in presence of optimal control and without control for a Un-notified cases with strain-1, b Un-notified cases with strain-2, $\mathbf{c}$ Notified cases with strain-1, $\mathbf{d}$ Notified cases with strain- 1 and $\mathbf{e}$ infected camels. The optimal control strategy is the combination of $U_{1}(t)$ and $U_{4}(t)$ 
(a)

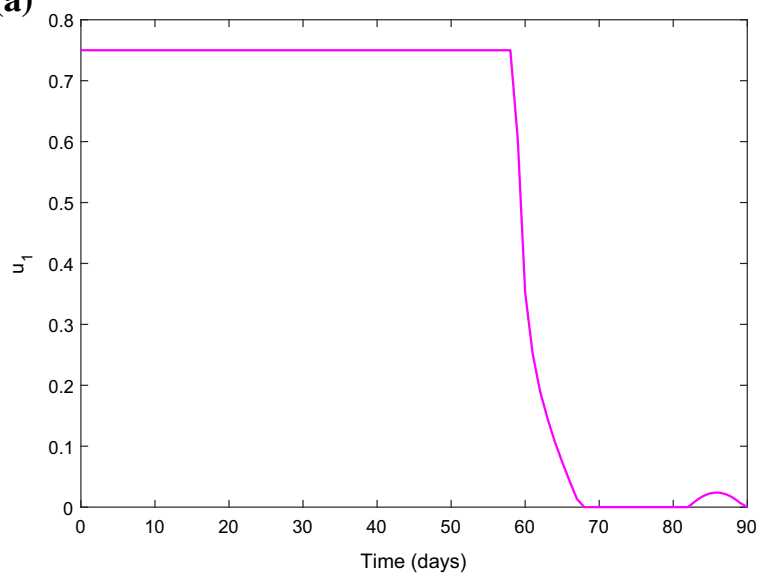

(b)

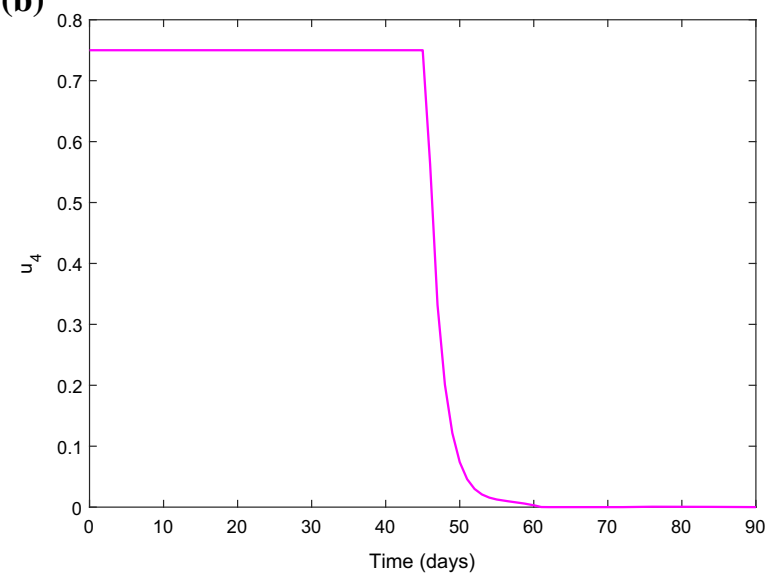

Fig. 5 Time evolution of optimal control profiles $\mathbf{a} u_{1}(t)$ and $\mathbf{b} u_{4}(t)$

strategy. This strategy is the most cost effective in terms of ACER and IAR.

\section{Discussion and conclusion}

In this paper, we introduce a general autonomous model that considers human and camel populations and probe further to understand the effect of optimal control strategies on the MERS-CoV infected populations. We mathematically analyze the single strain model to derive the basic reproduction number $R_{01}$ for the model and show that the disease-free equilibrium of the proposed model is globally asymptotically stable if $R_{01}<R_{01}^{c}<1$. Further, we prove the existence of backward bifurcation phenomenon for the single strain version of the proposed model under some parametric conditions. We also numerically showed the existence of backward bifurcation, see Fig. 2. The basic reproduction number $\left(R_{0}\right)$ of the full model is obtained and we numerically depicted the bistability even if $R_{0}<1$, see Fig. 3. Thus, the existence of bistability may be an inherent property of the MERS-CoV transmission dynamics. This phenomenon also indicates that the control interventions will require additional efforts to eradicate the disease from the community. To find the most influential parameters on $R_{0}$, we performed the normalized sensitivity analysis. Results indicate that, transmission rate from strain-1 infected humans to susceptible camels and transmission rate from camels to susceptible humans cam be decreased by using personal protection measures to reduce the epidemic potential. Additionally, the recovery rate of un-notified infected with strain- 1 should be increased to reduce the $R_{0}$ below unity. This can be achieved by finding and treating un-notified infected humans with strain-1.

Time dependent intervention strategies can be implemented to curtail a disease on a finite time interval. Using optimal control analysis, we further investigated the effects of intervention strategies on infected human and camel populations in the model (1). We consider four types of control, i.e. effect of self-protection measures by susceptible humans, the isolation of notified humans infected with strain-1 or strain- 2 and isolation of infected camels. Optimal control policy suggests that the combination of self-protection measures by susceptible humans and isolation of infected camels give cost-effective and efficient results for reducing disease prevalence. From Fig. 4, it can be observed that this combination strategy significantly reduces MERS$\mathrm{CoV}$ cases. Finally, we conclude that cutting the transmission cycle between camels and humans is the key factor to eradicate the disease from the community.

Acknowledgements The authors are grateful to the anonymous referees for their careful reading, valuable comments and helpful suggestions, which have helped to improve the standard of this work. Research of Indrajit Ghosh is supported by National Board for Higher Mathematics (NBHM) postdoctoral fellowship (Ref. No: 0204/3/2020/R \& D-II/2458). Sk Shahid Nadim receives funding from Council of Scientific \& Indus- 
trial Research (CSIR) as a senior research fellowship (Grant No: 09/093(0172)/2016/EMR-I), Government of India, New Delhi.

\section{Compliance with ethical standards}

Conflict of interest The authors declare that they have no conflict of interest.

\section{References}

1. Abate, A., Tiwari, A., Sastry, S.: Box invariance in biologically-inspired dynamical systems. Automatica 45(7), 1601-1610 (2009)

2. Assiri, A., McGeer, A., Perl, T.M., Price, C.S., Al Rabeeah, A.A., Cummings, D.A., Alabdullatif, Z.N., Assad, M., Almulhim, A., Makhdoom, H., et al.: Hospital outbreak of middle east respiratory syndrome coronavirus. N. Engl. J. Med. 369(5), 407-416 (2013)

3. Brauer, F., Castillo-Chavez, C., Mubayi, A., Towers, S.: Some models for epidemics of vector-transmitted diseases. Infect. Disease Modell. 1(1), 79-87 (2016)

4. Castillo-Chavez, C., Song, B.: Dynamical models of tuberculosis and their applications. Math. Biosci. Eng. 1(2), 361404 (2004)

5. Chan, R.W., Hemida, M.G., Kayali, G., Chu, D.K., Poon, L.L., Alnaeem, A., Ali, M.A., Tao, K.P., Ng, H.Y., Chan, M.C., et al.: Tropism and replication of middle east respiratory syndrome coronavirus from dromedary camels in the human respiratory tract: an in-vitro and ex-vivo study. Lancet Respiratory Med. 2(10), 813-822 (2014)

6. Chowell, G., Blumberg, S., Simonsen, L., Miller, M.A., Viboud, C.: Synthesizing data and models for the spread of mers-cov, 2013: key role of index cases and hospital transmission. Epidemics 9, 40-51 (2014)

7. Chung, J.S., Ling, M.L., Seto, W.H., Ang, B.S.P., Tambyah, P.A.: Debate on mers-cov respiratory precautions: surgical mask or $n 95$ respirators? Singapore Med. J. 55(6), 294 (2014)

8. Cotten, M., Watson, S.J., Kellam, P., Al-Rabeeah, A.A., Makhdoom, H.Q., Assiri, A., Al-Tawfiq, J.A., Alhakeem, R.F., Madani, H., AlRabiah, F.A., et al.: Transmission and evolution of the middle east respiratory syndrome coronavirus in saudi arabia: a descriptive genomic study. The Lancet 382(9909), 1993-2002 (2013)

9. EMPRES (2019) Empres. http://empres-i.fao.org/eipws3g/

10. Funk, A.L., Goutard, F.L., Miguel, E., Bourgarel, M., Chevalier, V., Faye, B., Peiris, J., Van Kerkhove, M.D., Roger, F.L.: Mers-cov at the animal-human interface: inputs on exposure pathways from an expert-opinion elicitation. Front. Vet. Sci. 3, 88 (2016)

11. Garba, S.M., Gumel, A.B., Bakar, M.A.: Backward bifurcations in dengue transmission dynamics. Math. Biosci. 215(1), 11-25 (2008)

12. Ghosh, I., Sardar, T., Chattopadhyay, J.: A mathematical study to control visceral leishmaniasis: an application to south sudan. Bull. Math. Biol. 79(5), 1100-1134 (2017)
13. Gumel, A.: Causes of backward bifurcations in some epidemiological models. J. Math. Anal. Appl. 395(1), 355-365 (2012)

14. Hsieh, Y.H.: 2015 middle east respiratory syndrome coronavirus (mers-cov) nosocomial outbreak in south korea: insights from modeling. PeerJ 3, e1505 (2015)

15. Ithete, N.L., Stoffberg, S., Corman, V.M., Cottontail, V.M., Richards, L.R., Schoeman, M.C., Drosten, C., Drexler, J.F., Preiser, W.: Close relative of human middle east respiratory syndrome coronavirus in bat, south africa. Emerg. Infect. Dis. 19(10), 1697 (2013)

16. Jones, J.H.: Notes on r0. Department of Anthropological Sciences, Califonia (2007)

17. Kamgang, J.C., Sallet, G.: Computation of threshold conditions for epidemiological models and global stability of the disease-free equilibrium (dfe). Math. Biosci. 213(1), 1-12 (2008)

18. Lee, D., Masud, M., Kim, B.N., Oh, C.: Optimal control analysis for the mers-cov outbreak: South korea perspectives. J. Korean Soc. Indus. Appl. Math. 21(3), 143-154 (2017)

19. Lee, J., Chowell, G., Jung, E.: A dynamic compartmental model for the middle east respiratory syndrome outbreak in the republic of korea: a retrospective analysis on control interventions and superspreading events. J. Theor. Biol. 408, 118-126 (2016)

20. Lenhart, S., Workman, J.T.: Optimal control applied to biological models. CRC Press, USA (2007)

21. Lin, Q., Chiu, A.P., Zhao, S., He, D.: Modeling the spread of middle east respiratory syndrome coronavirus in saudi arabia. Stat. Methods Med. Res. 27(7), 1968-1978 (2018)

22. Lukes, D.L., DL, L.: Differential Equations: Classical to Controlled. Academic Press, Singapore (1982)

23. Martcheva, M.: Methods for deriving necessary and sufficient conditions for backward bifurcation. J. Biol. Dyn. 13(1), 538-566 (2019)

24. Nadim, S.S., Chattopadhyay, J.: Occurrence of backward bifurcation and prediction of disease transmission with imperfect lockdown: A case study on covid-19. Chaos, Solitons and Fractals 140(110), 163 (2020)

25. Okosun, K.O., Rachid, O., Marcus, N.: Optimal control strategies and cost-effectiveness analysis of a malaria model. BioSystems 111(2), 83-101 (2013)

26. Poletto, C., Colizza, V., Boëlle, P.Y.: Quantifying spatiotemporal heterogeneity of mers-cov transmission in the middle east region: a combined modelling approach. Epidemics 15, $1-9$ (2016)

27. Reusken, C.B., Haagmans, B.L., Müller, M.A., Gutierrez, C., Godeke, G.J., Meyer, B., Muth, D., Raj, V.S., SmitsDe Vries, L., Corman, V.M., et al.: Middle east respiratory syndrome coronavirus neutralising serum antibodies in dromedary camels: a comparative serological study. Lancet. Infect. Dis 13(10), 859-866 (2013)

28. Sabir, J.S., Lam, T.T.Y., Ahmed, M.M., Li, L., Shen, Y., Abo-Aba, S.E., Qureshi, M.I., Abu-Zeid, M., Zhang, Y., Khiyami, M.A., et al.: Co-circulation of three camel coronavirus species and recombination of mers-covs in saudi arabia. Science 351(6268), 81-84 (2016)

29. Sardar, T., Ghosh, I., Rodó, X., Chattopadhyay, J.: A realistic two-strain model for mers-cov infection uncovers the 
high risk for epidemic propagation. PLoS Neglected Tropical Diseases 14(2), e0008,065 (2020)

30. Wang, J., Huang, Z., Wu, Z., Cao, J., Shen, H.: Extended dissipative control for singularly perturbed pdt switched systems and its application. IEEE Trans. Circuits Syst. I Regul. Pap. 67(12), 5281-5289 (2020a)

31. Wang, Y., Hu, X., Shi, K., Song, X., Shen, H.: Networkbased passive estimation for switched complex dynamical networks under persistent dwell-time with limited signals. J. Franklin Inst. 357(15), 921-936 (2020b)

32. Wangari, I.M., Stone, L.: Backward bifurcation and hysteresis in models of recurrent tuberculosis. PloS one 13(3), e0194,256 (2018)

33. WHO (2019) World health organization. https://www.who. int/csr/don/archive/disease/coronavirus_infections/en/

34. WLE (2019) world life expectancy. https://www.worldlifeexpectancy.com/ mammal-life-expectancy-dromedary

35. WPO (2019) Saudi arabia population. http://worldpopulationreview.com/countries/ saudi-arabia-population/

36. Zaki, A.M., Van Boheemen, S., Bestebroer, T.M., Osterhaus, A.D., Fouchier, R.A.: Isolation of a novel coronavirus from a man with pneumonia in saudi arabia. N. Engl. J. Med. 367(19), 1814-1820 (2012)
37. Zhang, B., Zheng, W.X., Xu, S.: Filtering of markovian jump delay systems based on a new performance index. IEEE Trans. Circuits Syst. I Regul. Pap. 60(5), 1250-1263 (2013)

38. Zumla, A., Hui, D.S., Perlman, S.: Middle east respiratory syndrome. The Lancet 386(9997), 995-1007 (2015)

Publisher's Note Springer Nature remains neutral with regard to jurisdictional claims in published maps and institutional affiliations. 